\title{
Neutron measurements from antineutrino hydrocarbon reactions
}

M. Elkins, ${ }^{1,}{ }^{*}$ T. Cai, ${ }^{2}$ J. Chaves, ${ }^{3}$ J. Kleykamp, ${ }^{2}$ F. Akbar, ${ }^{4}$ L. Albin, ${ }^{1}$ L. Aliagaa, ${ }^{5}$ D. A. Andrade, ${ }^{7}$ M. V. Ascencio, ${ }^{6}$ A. Bashyal, ${ }^{8}$ L. Bellantoni, ${ }^{9}$ A. Bercellie, ${ }^{2}$ M. Betancourt, ${ }^{9}$ A. Bodek, ${ }^{2}$ A. Bravar, ${ }^{10}$ H. Budd,${ }^{2}$ G. Caceres, ${ }^{11}$ M. F. Carneiro, ${ }^{8}$ D. Coplowe, ${ }^{12}$ H. da Motta, ${ }^{11}$ S. A. Dytman, ${ }^{13}$ G. A. Díaz,${ }^{2,6}$ J. Felix, ${ }^{7}$ L. Fields,${ }^{9,14}$ A. Filkins, ${ }^{5}$ R. Fine, ${ }^{2}$ N. Fiza, ${ }^{15}$ A. M. Gago, ${ }^{6}$ R. Galindo, ${ }^{16}$ A. Ghosh,${ }^{16,11}$ R. Gran, ${ }^{1}$ J. Y. Han, ${ }^{13}$ A. Habig, ${ }^{1}$ D. A. Harris, ${ }^{9}$ S. Henry, ${ }^{2}$ S. Jena, ${ }^{15}$ D. Jena, ${ }^{9}$ M. Kordosky, ${ }^{5}$ D. Last, ${ }^{3}$ T. Le, ${ }^{17,18}$ J. R. Leistico, ${ }^{1}$ A. G. Lopez, ${ }^{1}$ A. Lovlein, ${ }^{1}$ X.-G. Lu, ${ }^{12}$ E. Maher, ${ }^{19}$ S. Manly, ${ }^{2}$ W. A. Mann, ${ }^{17}$ C. M. Marshall, ${ }^{2, \uparrow}$ C. Mauger, ${ }^{3}$ A. M. McGowan, ${ }^{2}$ K. S. McFarland, ${ }^{2,9}$ B. Messerly, ${ }^{13}$ J. Miller, ${ }^{16}$ J. G. Morfín, ${ }^{9}$ J. Mousseau ${ }^{20, \$}$ D. Naples, ${ }^{13}$ J. K. Nelson, ${ }^{5}$ C. Nguyen, ${ }^{20}$ A. Norrick, ${ }^{9,5}$ Nuruzzaman, ${ }^{18,16}$ A. Olivier, ${ }^{2}$ V. Paolone, ${ }^{13}$ G. N. Perdue,${ }^{9,2}$ M. A. Ramírez, ${ }^{7}$ R. D. Ransome ${ }^{18}$ H. Ray, ${ }^{20}$ D. Rimal, ${ }^{20}$ P. A. Rodrigues, ${ }^{12,21,2}$ D. Ruterbories, ${ }^{2}$ H. Schellman, ${ }^{8,14}$ C. J. Solano Salinas, ${ }^{22}$ H. Su, ${ }^{13}$ V. S. Syrotenko, ${ }^{17}$ S. Sánchez Falero, ${ }^{6}$ E. Valencia, ${ }^{5,7}$ J. Wolcott, ${ }^{2,8}$ and B. Yaeggy ${ }^{16}$

\section{(MINERvA Collaboration)}

\author{
${ }^{1}$ Department of Physics, University of Minnesota-Duluth, Duluth, Minnesota 55812, USA \\ ${ }^{2}$ University of Rochester, Rochester, New York 14627 USA \\ ${ }^{3}$ University of Pennsylvania, Philadelphia, Pennsylvania 19104, USA \\ ${ }^{4}$ AMU Campus, Aligarh, Uttar Pradesh 202001, India \\ ${ }^{5}$ Department of Physics, College of William \& Mary, Williamsburg, Virginia 23187, USA \\ ${ }^{6}$ Sección Física, Departamento de Ciencias, Pontificia Universidad Católica del Perú, \\ Apartado 1761, Lima, Perú \\ ${ }^{7}$ Campus León y Campus Guanajuato, Universidad de Guanajuato, \\ Lascurain de Retana No. 5, Colonia Centro, Guanajuato 36000, Guanajuato México \\ ${ }^{8}$ Department of Physics, Oregon State University, Corvallis, Oregon 97331, USA \\ ${ }^{9}$ Fermi National Accelerator Laboratory, Batavia, Illinois 60510, USA \\ ${ }^{10}$ University of Geneva, 1211 Geneva 4, Switzerland \\ ${ }^{11}$ Centro Brasileiro de Pesquisas Físicas, Rua Dr. Xavier Sigaud 150, Urca, \\ Rio de Janeiro, Rio de Janeiro, 22290-180, Brazil \\ ${ }^{12}$ Oxford University, Department of Physics, Oxford, United Kingdom \\ ${ }^{13}$ Department of Physics and Astronomy, University of Pittsburgh, Pittsburgh, Pennsylvania 15260, USA \\ ${ }^{14}$ Northwestern University, Evanston, Illinois 60208, USA \\ ${ }^{15}$ Indian Institute of Science Education and Research - Mohali, \\ Knowledge city, Sector 81, SAS Nagar, Manauli PO 140306, India \\ ${ }^{16}$ Departamento de Física, Universidad Técnica Federico Santa María, \\ Avenida España 1680 Casilla 110-V, Valparaíso, Chile \\ ${ }^{17}$ Physics Department, Tufts University, Medford, Massachusetts 02155, USA \\ ${ }^{18}$ Rutgers, The State University of New Jersey, Piscataway, New Jersey 08854, USA \\ ${ }^{19}$ Massachusetts College of Liberal Arts, 375 Church Street, North Adams, Massachusetts 01247, USA \\ ${ }^{20}$ University of Florida, Department of Physics, Gainesville, Florida 32611, USA \\ ${ }^{21}$ University of Mississippi, Oxford, Mississippi 38677, USA \\ ${ }^{22}$ Universidad Nacional de Ingeniería, Apartado 31139, Lima, Perú
}

(Received 15 January 2019; published 5 September 2019)

\begin{abstract}
Charged-current antineutrino interactions on a hydrocarbon scintillator in the MINERvA detector are used to study activity from their final-state neutrons. To ensure that most of the neutrons are from the primary interaction, rather than hadronic reinteractions in the detector, the sample is limited to momentum transfers
\end{abstract}

\footnotetext{
Now at Iowa State University, Ames, Iowa 50011, USA.

${ }^{\dagger}$ Now at Lawrence Berkeley National Lab, Berkeley, California 94720, USA.

${ }^{*}$ Now at University of Michigan, Ann Arbor, Michigan 48109, USA.

${ }^{\S}$ Now at Tufts University, Medford, Massachusetts 02155, USA.
}

Published by the American Physical Society under the terms of the Creative Commons Attribution 4.0 International license. Further distribution of this work must maintain attribution to the author(s) and the published article's title, journal citation, and DOI. Funded by $S C O A P^{3}$. 
below $0.8 \mathrm{GeV} /$ c. From 16129 interactions, 15246 neutral particle candidates are observed. The reference simulation predicts $64 \%$ of these candidates are due to neutrons from the antineutrino interaction directly but also overpredicts the number of candidates by $15 \%$ overall. This discrepancy is beyond the standard uncertainty estimates for models of neutrino interactions and neutron propagation in the detector. We explore these two aspects of the models using the measured distributions for energy deposition, time of flight, position, and speed. We also use multiplicity distributions to evaluate the presence of a two-nucleon knockout process. These results provide critical new information toward a complete description of the hadronic final state of neutrino interactions, which is vital to neutrino oscillation experiments.

DOI: 10.1103/PhysRevD.100.052002

\section{INTRODUCTION}

Neutrons are the last essential (anti)neutrino-interaction final-state particle to have their number and energy distribution studied in detail. Neutrons play a special role in those oscillation measurements, which depend on comparing distortions of the antineutrino energy spectrum to the neutrino energy spectrum [1-6]. The antineutrino reactions' calorimetric response is heavily suppressed relative to the neutrino case because of the prevalence of neutrons, consistent with their generic role in hadron calorimetry [7]. The neutron energy deposits are not proportional to their kinetic energy and are not always observed at a location consistent with their initial trajectory from the interaction point. Sometimes, they are not observed at all because they escape the detector volume unseen, their interaction products are below detection threshold, or they thermalize without producing enough ionization to be reconstructed. In addition to energy determination, neutrons' presence in the final state also impacts event selection and background rejection for oscillation, interaction, and rare process analyses.

In this paper, we present the first direct measurements of the neutron content from neutron-rich antineutrino charged-current reactions. Neutrons with ten to hundreds of $\mathrm{MeV}$ kinetic energy are observed as they rescatter off hydrogen and carbon in the MINERvA detector. This study is done with a low three-momentum transfer sample $[8,9]$ but is otherwise inclusive with no selection on the number or type of hadrons in the final state. Low overall hadronic activity means that neutron activity is easily separated and likely to be due to neutrons from the original neutrino interaction rather than secondary hadronic reactions. The results are compared to the predictions of a full simulation that consists of a modified version of the GENIE neutrino event generator [10], a GEANT4 simulation of particle propagation in the detector material $[11,12]$, and a simulation of the calibrated response of our scintillator and electronics $[13,14]$.

The energy deposit, time, position, speed, and multiplicity distributions are sensitive to the details of neutron production in the initial reaction and to models for neutron scattering in the detector. When the neutrino reaction occurs in a nucleus, modeling the hadronic final state is complicated. Different charged-current weak-interaction processes produce different numbers of neutrons after the final-state lepton gains its charge. The charge-changing antineutrino quasielastic (QE) and two-particle two-hole $(2 p 2 h)$ process must turn at least one proton into a neutron. Often, the neutron has all of the hadronic final-state energy. In resonance production and deeply inelastic scattering (DIS), the charge can be exchanged with the resulting meson or the struck nucleon or quark, producing a higher number of neutrons per event than the equivalent neutrino case. These outcomes for carbon are summarized in Table I. For antineutrino reactions on hydrogen, the $2 p 2 h$ and finalstate interactions (FSI) do not occur; also, charged-current neutrino-hydrogen $\mathrm{QE}$ reactions are not possible.

Sensitivity to the mix of reaction types is reduced and indirect because the resulting hadrons will frequently reinteract on their way out of the nucleus. In event generators, these rescattering processes are referred to as FSI. Such reinteractions can be soft scatters that do not change the outgoing charge state, full charge exchange reactions where the energetic neutron becomes a proton or vice versa, and knockout reactions where multiple nucleons and mesons exit the nucleus. Calorimetric measurement of the interaction is affected, and the hadron topology also changes.

Neutrons from neutrino and antineutrino reactions have been measured before. The earliest technique was tagging the capture of thermal and "fast" (up to $10 \mathrm{MeV}$ ) neutrons, used from the very first neutrino observations [15] to the present and near future [16-19]. Higher-energy neutrons from neutrino reactions caused the most important $n p \rightarrow n p$ background to the discovery $[20,21]$ of weak neutral-current reactions in the freon-filled Gargamelle detector at CERN.

TABLE I. Typical number of free neutrons from chargedcurrent antineutrino reactions with carbon compared to neutrino reactions. In the extreme, FSI can break up the nucleus, releasing all the neutrons.

\begin{tabular}{lcc}
\hline \hline & \multicolumn{2}{c}{ Neutron content } \\
\cline { 2 - 3 } Process & Antineutrino & Neutrino \\
\hline QE & 1 & 0 \\
Resonance & 1 or 0 & 0 or 1 \\
$2 p 2 h$ & 2 or 1 & 1 or 0 \\
After FSI & 0 to 7 & 0 to 5 \\
\hline \hline
\end{tabular}


The collaboration made measurements of "associated neutrons" and used a cascade simulation [22] to translate that measurement into an estimate for neutron production from neutrinos interacting in the material upstream and so obtained the crucial constraint on the background. Similar studies were needed for follow-up neutral-current measurements including those with two liquid scintillator detectors [23-25] in the Brookhaven National Laboratory neutrino beam and the "dirt backgrounds" from MiniBooNE's measurement [26,27]. These neutrons' time and spatial distributions in the detector were measured and simulated in order to constrain and subtract backgrounds but were not correlated with simultaneous measurement of their original interaction in the material upstream of the detector. MiniBooNE's paper presents a comparison to a modern Monte Carlo simulation using the NuANCE neutrino event generator [28], and GEANT3 [29] using the GCALOR [30] option, and found that a $30 \%$ reduction of the neutron component was needed to describe the data.

Recently, a measurement [31] was presented by ArgoNeuT for neutrino-argon reactions which is similar to the one in this paper. The ArgoNeuT analysis studied low-energy photons produced by the deexcitation of the argon nucleus struck by the neutrino and by the deexcitation of argon nuclei struck by neutrons generated in the neutrino reaction. Unlike the MINERvA antineutrino data described in this paper, the neutron component is only half the sample, and the photons from deexcitation of the argon nucleus struck by the neutrino are evident in the spatial and multiplicity distributions. In the ArgoNeuT study, both components were simulated by FLUKA $[32,33]$. Another recent paper [34] breaks down the simulation of neutrino and antineutrino reactions with argon to provide details of the pathways to missing energy, especially via neutrons.

MINERvA has several advantages relevant to detecting neutrons from (anti)neutrino interactions. The 5.3 ton, fully active tracking volume is much larger than the neutroninteraction length of approximately $10 \mathrm{~cm}$ for neutron kinetic energies near $20 \mathrm{MeV}$. Larger volumes have been used (Super-K and MiniBooNE), but their Cerenkov technique has too high a threshold for detecting the protons scattered by neutrons. MINERvA's active volume is polystyrene $\left(\mathrm{C}_{8} \mathrm{H}_{8}\right)_{n}$, shortened to $\mathrm{CH}$ when used in nuclear and particle physics. For low-energy neutron detection, the hydrogen presents as significant a target as the carbon, despite a carbon nucleus having 12 nucleons. And MINERvA is sensitive down to a low threshold of $1 \mathrm{MeV}$ because it is an underground detector with low noise overall and well-constrained contributions from other sources of neutron production in the beam.

\section{MINERvA EXPERIMENT}

MINERvA is a dedicated neutrino-nucleus cross section experiment. Its goals are to make cross section measurements needed for neutrino oscillation experiments and to probe the environment of the nucleus, complementary to what the electron scattering community has accomplished. The experiment is located in the high-intensity NuMI neutrino beam at Fermilab.

The centerpiece of the detector [13] is a 5.3 ton fully active scintillator tracker with excellent calorimetric properties of its own and surrounded by additional electromagnetic and hadronic calorimeters. The experiment also has passive targets made of iron, lead, water, graphite, and helium, which enable the study of the A-dependence of neutrino and antineutrino reactions.

The tracker is built from planes of polystyrene scintillator strips. With Lexan sheets, epoxy, tape, and reflective titanium dioxide, the target consists of $8.2 \%, 88.5 \%$, and $2.5 \%$ hydrogen, carbon, and oxygen, respectively (by mass), plus small amounts of heavier nuclei. The strips are triangular in shape with a $3.3 \mathrm{~cm}$ base and $1.7 \mathrm{~cm}$ height and up to $245 \mathrm{~cm}$ length. The strips are nested with alternating orientation to make $1.7 \mathrm{~cm}$ thick planes. With this arrangement, ionization activity in the plane is split between strips, and tracking resolution is improved. An entire plane is a hexagon containing 127 strips and one module consists of two planes. The second plane in one module is oriented with the strips vertical, producing an $\mathrm{X}$-coordinate of the observed energy deposits. The plane in front of it is rotated $60 \mathrm{deg}$ one way to form a $\mathrm{U}$ coordinate or the other way to form a $\mathrm{V}$ coordinate. The resulting modules are themselves alternated to produce repeating UX, VX sets of planes, with the detector $\mathrm{Z}$ axis running normal to these planes. The MINOS Near Detector [35] is located $2 \mathrm{~m}$ downstream of MINERvA and measures the charge sign and momentum of muons selected in this analysis.

These data were obtained from the neutrinos at the main injector beam [36] operating in antineutrino mode. The primary $120 \mathrm{GeV}$ proton beam interacts in a graphite target, producing pions and kaons. Two magnetic horns focus the negatively charged mesons toward a decay pipe, leading to an antineutrino spectrum that peaks near $3.0 \mathrm{GeV}$. In total, these data are from an exposure of $1.04 \times 10^{20}$ protons on target between November 2010 and February 2011. This beam configuration also produces neutrino charged-current interactions in the detector which are over $10 \%$ of the events in the antineutrino peak energy range used in this analysis. These are removed with high efficiency because their measured curvature in the MINOS Near Detector is the wrong direction.

The flux prediction is GEANT4 based [11,12] with central values and uncertainties adjusted [37] using thin-target hadron production data [38-41] and an in situ neutrinoelectron scattering constraint [42]. The design of this analysis is relatively insensitive to the resulting $8 \%$ to $10 \%$ uncertainties in the energy spectrum or the absolute flux.

\section{SIMULATION}

The reference simulation combines the GENIE 2.8.4 neutrino-interaction model [10] with modifications, the 
GEANT4 9.4.P2 particle transport model $[11,12]$ with modifications, a calibrated detector time and energy response model $[13,14]$, and an "overlay" of data events to reproduce the unrelated activity that might overlap in time with the simulated event.

\section{A. GENIE event generator}

GENIE's simulation of the charged-current quasielastic process is from Llewellyn Smith [43] with vector form factors parametrized as in Ref. [44] and a dipole form factor with axial mass of $0.99 \mathrm{GeV}$. A relativistic Fermi gas model [45] is implemented for interactions on carbon and other nuclei. The $\Delta$ and higher resonances are from Rein and Sehgal [46], with a nonresonant component added from the DIS model as the resonances are phased out from invariant mass $1.4<\mathrm{W}<2.0 \mathrm{GeV}$. The DIS cross section comes from the Bodek-Yang model [47], where the hadronic system [48] is produced using Koba, Nielsen, and Olesen (KNO) scaling [49] transitioning to PYTHIA [50] between 2.4 and $3.0 \mathrm{GeV}$.

Modifications are made to the above default GENIE 2.8.4 model. We refer to this set as MINERvA tune version 1.1 (MNvGENIE-v1.1). These modifications can also be applied to the default 2.12.6 version of GENIE. The QE process is modified to include a screening effect based on the random phase approximation (RPA) technique. The suppression is based on the calculations of Nieves and collaborators [51,52] for a Fermi-gas nucleus and implemented by reweighting GENIE QE events [53], including an uncertainty on the RPA screening derived from comparison to neutron capture data $[54,55]$. The uncertainty on the QE axial form factor is set to $9 \%$ following the analysis of Ref. [56]; additional uncertainty on the highest $Q^{2}$ component is not needed for this sample. Nonresonance pion production is reduced based on the reanalysis of bubble chamber neutrino data $[57,58]$. Coherent pion production with pion kinetic energy below $450 \mathrm{MeV}$ is also reduced based on analysis of MINERvA data [59,60] and consistent with the Berger-Sehgal [61] modifications of the original Rein-Sehgal model [62].

Of special interest is the interaction of the antineutrino with two nucleons, knocking them both out and leaving two holes $(2 p 2 h)$ in the nucleus. In this analysis, the base model for the $2 p 2 h$ component with no pion is from the IFIC Valencia group [52,63] implemented in GENIE [64]. This process is further enhanced in the region between the $\mathrm{QE}$ and $\Delta$ resonance components based on a fit [65] to the reconstructed neutrino data presented in Ref. [9]. In all but the last figure in this paper, the error band includes an uncertainty on this fit that varies the fraction of reactions on $p n$ and $p p$ initial states. This enhancement has been applied to this analysis and successfully describes a wide variety of data for other MINERvA observables [8,66-70].

Reinteractions of nucleons and mesons (FSI) as they exit the target nucleus are the other important mechanism for neutron production. The GENIE simulation of final-state reinteractions of hadrons leaving the nucleus is a parametrized, effective cascade model which is called "hA," short for hadron-nucleus interaction. The model steps hadrons through a nucleus with its associated radius and nuclear density function. The hadron's mean free path is then determined from tabulated hadron-proton and hadronneutron cross sections from SAID [71]. The resulting probability of interacting within the nucleus is high, $73 \%$ for a neutron from a $3 \mathrm{GeV}$ quasielastic event in carbon and $88 \%$ in iron. Because the code originated with the MINOS experiment [35], when an interaction is specified, the fates (absorption, pion production, knockout, charge exchange, and elastic scatter) are chosen according to their proportions for iron. In-medium effects that might relatively favor particular fates in carbon or lead are not included in this original version; nor is multiple scattering. When one interaction occurs, the number of outgoing nucleons is drawn from a distribution that favors single nucleon, deuteron, and alpha states but allows a chance for complete breakup.

Compared to the cross sections for the initial reactions, the simulation of FSI differs the most among different event generators. The GENIE FSI model produces more low-energy nucleons than other commonly used neutrino event generators. Figure 1 shows the energy and multiplicity spectra for a newer (2.12.10) but equivalent version of GENIE with its models and modifications configured for the MNVGENIEV1.1 tune. It is compared to two other common generators NEUT [72,73] and NUWRO [74], produced using the NUISANCE framework [75]. The inputs are monoenergetic $3 \mathrm{GeV}$ antineutrinos on a $\mathrm{CH}$ target, and the $q_{3}<0.8 \mathrm{GeV}$ selection is made. Neutrons below $2 \mathrm{MeV}$ are not included in the kinetic energy distribution. Appropriate to this analysis, neutrons below $10 \mathrm{MeV}$ are not included in the multiplicity distribution. Below $25 \mathrm{MeV}$, the NEUT generator stops any reinteraction process (cross section is set to zero), so those neutrons are placed outside the nucleus rather than producing yet more and lower-energy neutrons. In contrast, above $50 \mathrm{MeV}$, each generator is using similar models for the primary production of neutrons before FSI and their predictions for the neutrons' kinetic energy spectra converge.

\section{B. GEANT4 neutron propagation model}

Particles in the final state are passed to GEANT4 9.4.P2 with the Bertini cascade option (QGSP_BERT), which propagates them through a model of the MINERvA detector geometry and materials. Uncertainties in the charged hadron-interaction cross section in the scintillator are implemented using a reweighting scheme and an uncertainty of $\pm 10 \%$ based on our analysis of this version of GEANT and data for protons [76-83] and pions [84-88]. Confidence in the proton and pion response at these energies also comes from analysis of calorimetric data taken with MINERvA detector elements in a test beam at 

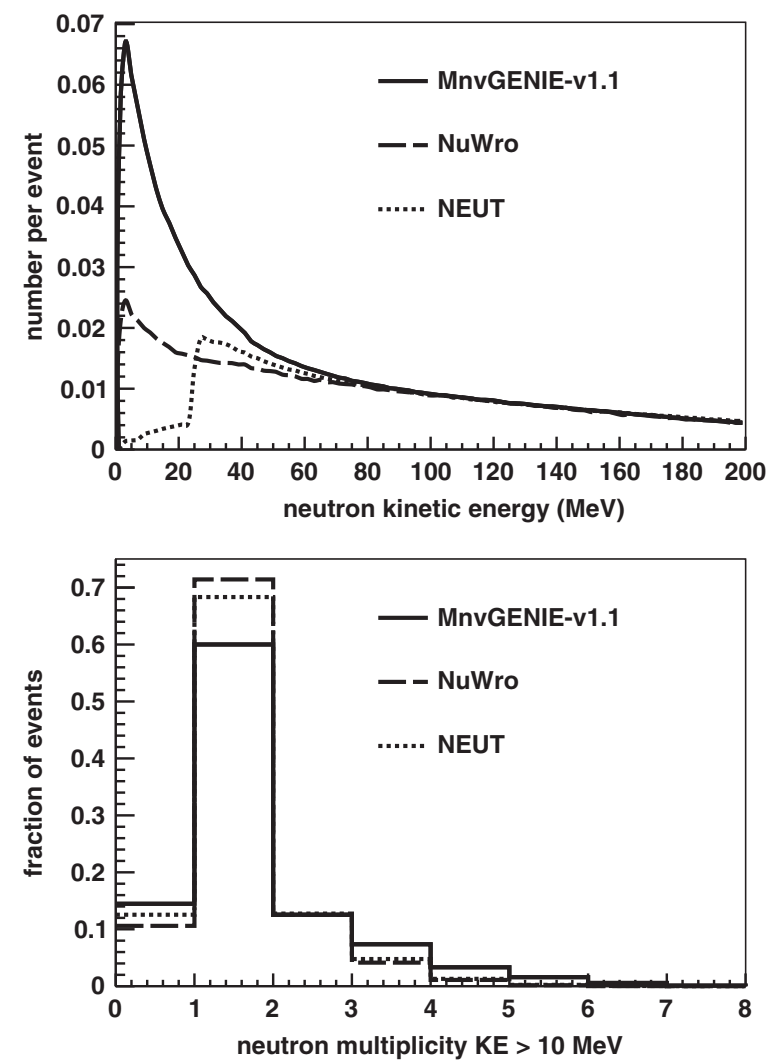

FIG. 1. Neutron energy spectra (upper) and multiplicity (lower) from three popular neutrino event generators for $3 \mathrm{GeV}$ antineutrinos interacting in $\mathrm{CH}$. There exists a wide range of predictions, especially for the lowest-energy component of this spectrum (see the text for a description).

Fermilab [14]. The charged hadron scattering cross sections in the detector are not major sources of uncertainty. The same study found the neutron inelastic scattering on nuclei also models the data [89-91] at the 10\% level, but the total cross section (elastic + inelastic) for this version does not describe the Abfalterer data [92].

The energy dependence of the total cross section for neutrons in GEANT4 has been improved since this older version. We have compared this version with a late 2016 release (v10.2.p2) to understand changes in the neutroninteraction code. The new versions now match the Abfalterer et al. high-precision neutron scattering total cross sections [92] on carbon and hydrogen from 5.2 to $560 \mathrm{MeV}$. Using the same reweighting tools as to evaluate hadron systematic uncertainties, the older GEANT4 cross section is changed to represent these same data. After making this correction, we assign uncertainties of $25 \%$ below $10 \mathrm{MeV}, 20 \%$ from $10 \mathrm{MeV}$ to $25 \mathrm{MeV}$, and $15 \%$ above $25 \mathrm{MeV}$. These are applied to the total cross section but are larger than the remaining discrepancy with the data. In this analysis, they play the effective role of uncertainties on the elastic vs inelastic components.

In principle, another uncertainty arises from the outcomes of the neutron-hydrogen and neutron-carbon interactions. The Abfalterer et al. total cross section includes elastic scatters that deflect the neutron as little as $0.12 \mathrm{deg}$, which involve sub-keV scale energy transfers. Thus, the GEANT4 model also makes a prediction for the fraction of scatters that are above and below our experimental threshold. The accuracy of this feature of the prediction is not well constrained by available data.

A second, prominent feature of the GEANT4 neutronscattering model is the production of deexcitation photons following neutron-carbon reactions. Such photons Compton scatter and account for 50\% of the neutron candidates that originate from a GENIE neutron. The simulation predicts the rest are from protons and up to $5 \%$ from nuclear fragments. For comparison, three recent accountings of neutron induced activity are presented in Refs. [31,34,93] for argon; the latter has especially detailed discussion of simulated activity.

GEANT4 provides an alternate, high precision neutron simulation (HP in the GEANT4 option names), originally designed for studies of fission reactions up to about $20 \mathrm{MeV}$. After accounting for the Abfalterer cross sections, the differences between the fully simulated HP configuration and the default configuration appear where the lowestenergy neutrons are substantial parts of the sample, especially near the interaction point. But these differences are modest, similar to the other uncertainties, and difficult to disentangle from the rest of the GEANT4 predicted response.

\section{Detector response}

The simulation also must produce energy deposits consistent with our calibrated scintillator and electronics response. The photoelectron yield and absolute energy scale are tuned using a comparison of data and simulated muons at near-normal incidence to the planes. Nonlinearities for energy deposits above minimum ionizing muons are accounted for via individual calibration of the digitizing electronics [13] and by the test beam calibration [14] of Birks's quenching in the scintillator.

The simulation reproduces the detailed response of light propagation in the scintillator bars and the photomultiplier tube, so that the simulated activity is reconstructed using identical steps as with the data. When a particle is fully tracked, every energy deposit's location is known in all three dimensions. In this case, the reconstruction estimates the effect of light reflection and attenuation in the scintillator strip and optical fiber and produces a more accurate estimate of the actual energy deposit. This is rarely possible for the neutron energy deposits in this analysis. When not a part of the track, the reconstruction approximates each energy deposit to happen at a position halfway along the scintillator bar. Geometrical fluctuations will therefore be present in the energy and timing distributions in both data and simulation. 
The width of the time distribution of digitized activity after light propagation depends on the photoelectron yield in the photomultiplier tube. The distribution used in the simulation is based on the observed distribution in data from fully tracked muons. Because averaging over the entire track yields a precise time and location for the passage of the muon through any one plane, the correlation of the fluctuations in time and the light yield in a single scintillator strip can be obtained directly, without resorting to individual photon modeling of the optical elements and photomultiplier tube response. The width of the time distribution is $10 \mathrm{~ns}$ for 1 to 2 photoelectrons and $3 \mathrm{~ns}$ for 6 to 12 (about 1 to $1.5 \mathrm{MeV}$ in a single strip) then approaches the electronics limit of $2.2 \mathrm{~ns}$. More detail on the nanosecond timing response and its use can be found in Ref. [94]. With geometry-based fluctuations, the final time resolution for neutron candidates is $4.5 \mathrm{~ns}$.

A subdominant contribution to neutron candidates in this analysis comes from other neutrino interactions inside and outside the detector, which produce their own neutrons and photons. They randomly happen at the same time as the charged-current reactions selected for this analysis. These "accidental" backgrounds are added directly to the simulation; $16 \mu$ s of activity from one pulse of the beam from data are selected randomly from the same months. This data activity is added on top of the activity from the simulated event. The resulting set of reconstructed times, locations, and energies are given to the same reconstruction algorithm. Thus, this background and its dependence on the intensity of the beam are reproduced. Using alternate selections to isolate four regions that are predicted to be high (greater than 70\%) in this particular background leads to the assignment of a $\pm 10 \%$ uncertainty applied to the simulation.

\section{Further modifications of the simulation}

The configurations within the simulation packages do not contain enough explicit uncertainties or tunable parameters to describe the data presented in this paper. Described in detail with the data in later sections, we will make two heuristic reductions in the number of neutron candidates in the simulation. One mimics a mismodeling of either or both the energy deposit spectrum and the number of deexcitation photons as simulated by GEANT4. The other reflects the wide range of predictions for low-energy neutrons from the neutrino interaction models suggested by Fig. 1. For this paper, the reductions allow us to quantitatively explore possible unknown effects, even though they do not represent a $\pm 1 \sigma$ uncertainty.

\section{EVENT SAMPLE AND NEUTRON SELECTION}

The sample of charged-current antineutrino events analyzed in this paper is the same inclusive sample from
Ref. [8] with reconstructed three-momentum transfer $q_{3}$ less than $0.8 \mathrm{GeV} / \mathrm{c}$. This sample has high neutron content but little other charged hadronic activity to complicate the analysis of neutron activity. This allows us to use a low threshold of $1.5 \mathrm{MeV}$ for neutron candidates.

\section{A. Selection of antineutrino events}

Charged-current antineutrino interactions originate in the scintillator in the active tracker fiducial region. The resulting $\mu^{+}$must be fully tracked to the end of the MINERvA detector and also reconstructed in the MINOS Near Detector [35] where its positive charge and momentum are analyzed. To ensure a region of good geometrical acceptance, we require $p_{\mu}>1.5 \mathrm{GeV} / \mathrm{c}$, and $\theta_{\mu}<25 \mathrm{deg}$. The selected reconstructed neutrino energy range is $2.0<E_{\nu}<6.0 \mathrm{GeV}$, so resulting data and simulated samples are the lower panels of Fig. 3 of Ref. [8] and also match the selection used for the related neutrino-mode analysis [9].

The muon energy and angle are combined with the observed hadronic energy deposits to form calorimetric estimates for the energy transfer $q_{0}$ (often called $\omega$ or $\nu$ by different groups in the literature), neutrino energy $E_{\nu}=q_{0}+$ $E_{\mu}$, square four-momentum transfer $-q^{2}=Q^{2}=2 E_{\nu}\left(E_{\mu}-\right.$ $\left.p_{\mu} \cos \theta_{\mu}\right)-M_{\mu}^{2}$, and the magnitude of the three-momentum transfer $q_{3}=\sqrt{Q^{2}+q_{0}^{2}}$ (often simply called $q$ or $|q|$ ). The selection is inclusive because only the magnitude of reconstructed $q_{3}<0.8 \mathrm{GeV} / \mathrm{c}$ enters the selection, not details of the number or type of hadrons observed.

We exploit the feature that this subsample can be divided into regions, hereafter called QE rich, dip, and $\Delta$ rich. The QE-rich region has little or no observed hadronic energy and is predicted to be mostly $2 p 2 h$ and QE. The $\Delta$-rich region has the most energy transfer and is predicted to be mostly resonance production and some $2 p 2 h$. The so-called dip region in between these two is a mix of all three processes but also has the highest predicted concentration of $2 p 2 h$ events. Again referring to the lower panels of Fig. 3 in Ref. [8], boundaries are formed between the QE-rich, dip-region, and $\Delta$-rich subsamples. The reconstructed "available energy" is an estimator for a quantity that includes proton and charged pion kinetic energy and the total energy of neutral pions, photons, and electrons produced by a neutrino-interaction model. The latter, built from the model prediction for each generated event, explicitly does not include kinetic energy of neutrons nor the energy cost to remove nucleons from the nucleus, so it is always lower than the true energy transfer and for some antineutrino reactions will be zero. The boundaries are at reconstructed available energy of 0.06 and $0.12 \mathrm{GeV}$ for $0.0<q_{3}<0.4 \mathrm{GeV} / \mathrm{c}$ and 0.08 and $0.18 \mathrm{GeV}$ for $0.4<q_{3}<0.8 \mathrm{GeV} / \mathrm{c}$. For brevity, many distributions in this paper are not divided this way and are presented as two $q_{3}$ regions. 


\section{B. Selection of neutral particle induced candidates}

Because the sample is limited to $q_{3}<0.8 \mathrm{GeV} / \mathrm{c}$, charged hadron activity is small and remains localized to the interaction point. The rest of the detector should have no hadronic activity except neutrons and photons, which this analysis considers signal. There are two backgrounds to neutron candidates to minimize: the "muon background" from electrons and bremsstrahlung photons and the "accidental background" from activity induced by the neutrino beam or cosmic rays and unrelated to the antineutrino reaction being analyzed.

Analysis of hadronic activity is done with reconstructed objects formed of "clusters" of energy deposits in one or multiple adjacent strips within the same plane. Each cluster is assigned a calibrated energy based on the sum of the individual energy deposits. A cluster also has a twodimensional position, one from the location of the plane in the detector and one from estimating the transverse position in its plane based on energy-weighted average positions of activity in multiple strips. The calibrated time is the weighted average of hit times, taking into account the measured correlation between the number of photoelectrons and the width of the timing distribution of single strip activity from muons in data.

The criteria for spatially isolated clusters are designed to exclude four overlapping volumes in spatial proximity to the muon activity, the interaction point, other charged hadron activity, or to the outer boundary of the detector. An event display of the X-coordinate activity simulated event, Fig. 2, summarizes how activity in the first three categories is rejected and a single cluster neutron candidate is observed.

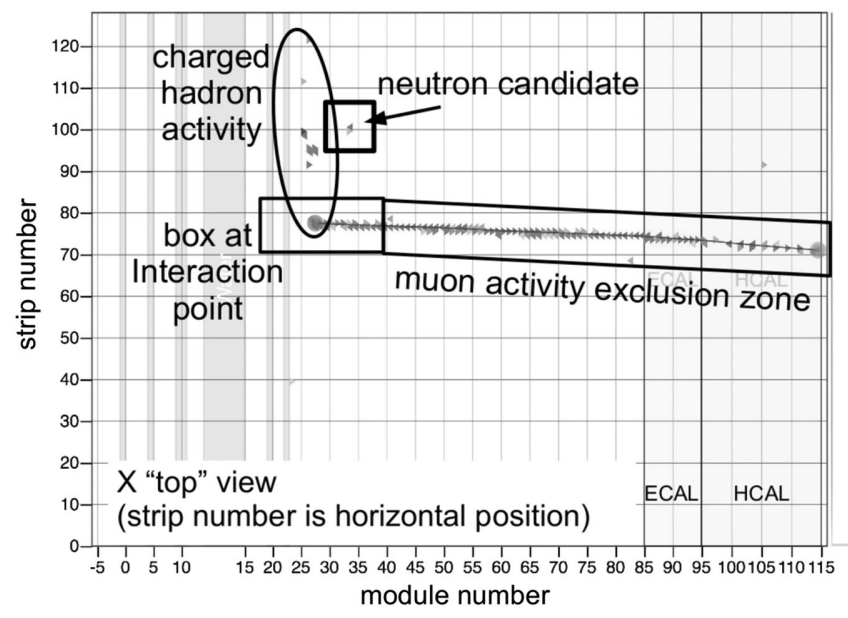

FIG. 2. Event display of a simulated event illustrating geometrical selections to avoid activity near the muon, event interaction point, and other charged hadron activity (a $\pi^{-}$in this simulated event) with the remaining activity promoted to a neutron candidate. The aspect ratio for this figure exaggerates the transverse dimension by almost a factor of 2 in order to emphasize the detail. Activity from the $\pi^{-}$in adjacent $U$ and $V$ planes near the interaction point is not shown.
Additional energy threshold and timing cuts complete the selection. Remaining clusters that are near each other, indicating they may be caused by the same neutral particle, are combined into multicluster candidates. The simulation is used to evaluate the effectiveness of these selections at reducing the muon and accidental backgrounds.

\section{Muon exclusion zone}

The muon itself is fully tracked for all events in this sample, and most clusters are already assigned to the reconstructed track. Additional activity near the muon track is very likely caused by photons from bremsstrahlung and knock-on electrons (delta rays). From one module upstream of the interaction point to the back of the detector, we exclude clusters within $17 \mathrm{~cm}$ (about ten strips) of the muon from consideration, about one mean free path for photons with energy of a few mega-electron-volts. The simulation predicts that antimuon induced activity accounts for $90 \%$ of what would otherwise be candidates in this zone, but the simulation also underpredicts the data by $12 \%$. This exclusion reduces the muon-induced background by a factor of 10 .

This zone is increased to $24 \mathrm{~cm}$ starting 20 modules downstream from the interaction point, effectively a simplified implementation of an exclusion cone. The additional volume is predicted to be $65 \%$ muon activity and 3\% accidental backgrounds, and excluding it further reduces the muon background by another factor of 2 . The simulation describes the activity in this outer zone well, contributing 3.5\% fewer clusters than data. We assign twice this difference as the uncertainty on the muon contribution to the remaining selected clusters, which is negligible for this analysis.

\section{Interaction point exclusion zone box}

Charged hadrons will produce activity near the neutrinointeraction point and the start of the muon track. The granularity of the detector imposes limits on separating muon, hadron, photon, and cross-talk activity when some or all of it fails to meet tracking criteria. This analysis follows our past analysis strategy $[68,70,95,96]$ to avoid this region and its complicated systematic uncertainties for a primary analysis. The neutrino-nteraction model is most challenged when asked to predict activity near the interaction point. This creates uncertain neutron detection efficiency effects and is deferred for future investigations.

Clusters of activity within a transverse "box" around this point are excluded from further consideration. Because of the three $\mathrm{X}, \mathrm{U}, \mathrm{V}$ orientations, the box has a pinwheel shape but can be defined and coded simply when threedimensional reconstruction is not available. This exclusion is for clusters within $17 \mathrm{~cm}$ transverse from a horizontal line parallel to the detector $\mathrm{Z}$ axis through the start of the muon track from ten modules upstream to fifteen modules downstream. In the downstream direction, it usually overlaps 
significantly with the muon exclusion zone. This region remains rich with neutron activity and may be explored in future neutron analyses.

\section{Charged particle exclusion zone}

Some charged hadrons travel outside the interaction point exclusion zone. A spatial algorithm considers seed clusters near the interaction point and adds additional clusters to the total charged particle activity if they are nearby. This algorithm will follow such spatially connected activity arbitrarily far from the interaction point. This includes many $\pi^{-}$and a few protons that are fully tracked but also activity from reinteractions in the detector that do not satisfy stricter requirements to form or extend a track object. This is the first full deployment of such an algorithm in a MINERvA analysis.

The procedure starts with a list of clusters that are inconsistent with cross-talk based on their energy and pixel location relative to other observed energy deposits within the same 64-pixel photomultiplier tube. Then, the two-dimensional distance to the neutrino-interaction point is formed. If at least one cluster is found within $250 \mathrm{~mm}$, the list is searched again, and any cluster within $100 \mathrm{~mm}$ of the first one is excluded from further consideration. Additional clusters are compared until no other cluster satisfies the $100 \mathrm{~mm}$ requirement with any prior cluster. Then, it iterates five more times with the original $250 \mathrm{~mm}$ cluster test, which may identify additional hadrons going off in completely different directions.

For the many clusters near the interaction point, this procedure is largely redundant with the vertex exclusion box. However, it will follow spatially connected energy well outside the other exclusion zones. This trivially includes energy that was already part of a hadron track. It also includes energy produced when a hadron reinteracts, creating additional untracked energy, and also hadrons from the interaction point that did not satisfy the stricter requirements to form a track object.

The performance of this selection can be illustrated by the situations that lead to a neutron candidate attributed to a $\pi^{-}$from GENIE, which is one component of the signal and background that remain after all selections and summarized in Table II. After inspecting the GEANT4 trajectory information, $85 \%$ of those neutron candidates from $\pi^{-}$were caused by secondary neutrons, photons from nuclear deexcitation, and photons from the decay of neutral pions from charge exchange in the detector. The other $15 \%$ were not incorporated into the charged particle exclusion zone because some activity was highly transverse to the detector. There is such a simulated $\pi^{-}$in the example in Fig. 2. Unlike this example, there is sometimes a cluster at a high angle that did not meet the $100 \mathrm{~mm}$ tolerance to extend the exclusion zone. In total, these situations account for only $3 \%$ of the total candidate sample.
TABLE II. Particle from the GENIE simulation leaving the nucleus that caused the simulated candidates, showing the characteristics of the signal and background. Candidates attributed to proton and $\pi^{-}$from GENIE are largely caused by secondary neutrons. The lower part of the table shows the number of selected events and neutron candidates per event (cand/evt) for this data and simulated exposure. Compared to the data, the simulation has an overprediction of candidates caused by neutrons.

\begin{tabular}{lcc}
\hline \hline & \multicolumn{2}{c}{ 3-momentum transfer } \\
\cline { 2 - 3 } GENIE particle & $0<q_{3}<0.4$ & $0.4<q_{3}<0.8$ \\
\hline Neutron & $78.1 \%$ & $60.8 \%$ \\
Proton & $0.4 \%$ & $1.6 \%$ \\
$\pi^{-}$ & $12.3 \%$ & $22.8 \%$ \\
$\pi^{0}$ & $2.6 \%$ & $10.3 \%$ \\
Muon & $4.0 \%$ & $2.4 \%$ \\
Data overlay & $2.1 \%$ & $1.4 \%$ \\
Other & $0.6 \%$ & $0.6 \%$ \\
& & \\
Events sim & 4499 & 11651 \\
Events data & 4897 & 11263 \\
Cand/evt sim & 0.647 & 1.284 \\
Cand/evt data & 0.584 & 1.103 \\
\hline \hline
\end{tabular}

\section{Edge of detector, timing, and energy}

Activity at the edges of the detector is excluded as follows: the first 20 planes and veto wall upstream, the last 10 planes in the downstream hadronic calorimeter, and the entire outer detector hadronic calorimeter. Most clusters are naturally found in the inner tracker region because it is near to the interaction point, has the largest fraction of the active scintillator in the detector, and the scintillator has the highest hydrogen content of all the detector materials.

A few additional selections reduce the already small accidental background. Clusters not yet excluded must be within a time window from $20 \mathrm{~ns}$ before to $35 \mathrm{~ns}$ after the interaction time $t_{0}$ determined largely from the muon track timing information. The clusters must also have at least $1.5 \mathrm{MeV}$ of energy. The accidental background overtakes the predicted signal processes at cluster energies below 1.2 MeV. This energy cut also eliminates photomultiplier tube cross-talk effects in both selected data and simulated events. This version of the accidental background overlay technique is not perfect. For this early version, it is checked against the data for accidental-rich subsamples, leading to an uncertainty of $10 \%$. This conservative uncertainty has a negligible impact on the results.

\section{Aggregating spatially nearby clusters into candidates}

Some isolated clusters can be spatially connected. Two clusters within three modules of each other are merged into one neutron candidate. This merging continues if additional clusters satisfy this requirement. Because there is so little hadronic activity in these low $q_{3}$ subsamples, this simple 
requirement is effective. In this sample, $62 \%$ are candidates made of a single cluster, $18 \%$ are made of exactly two clusters; the simulation predicts $61 \%$ and $19 \%$, respectively.

\section{Signal and background}

Neutrons that exit the nucleus where the neutrino interaction occurred are of the most interest. They are the aspect of the interaction model that has never before been directly tested. When referring to the GENIE simulation of this component, including direct production and via FSI, we will call them GENIE neutrons. Protons and charged pions produce real secondary neutrons as they travel through the detector. These are an irreducible background, and no attempt is made to reject them. Table II quantifies the sources that cause the majority of neutron candidates.

Photons also produce energy deposits distant from the main parts of the event. Thus, the decay products of $\pi^{0}$ are an electromagnetic component of candidates. The $\pi^{-}$ entries in Table II include 14\% (so $1.7 \%$ and $3.2 \%$ of the respective column totals) which charge exchanged to $\pi^{0}$ in the detector. With such little hadronic energy in the final state, it is convenient to treat these also as irreducible backgrounds and make no selection to reduce them. Because of the $q_{3}$ selection, these photons are relatively low energy. MINERvA's $\gamma$ and $\pi^{0}$ selection algorithms $[67,97]$ have an efficiency of $15 \%$ because $\pi^{0}$ identification requires two reconstructed photon candidates and an effective threshold of $25 \mathrm{MeV}$ on the lower-energy photon. As will be seen, the electromagnetic component traveling at the speed of light will separate from slower neutrons in distributions that use time-of-flight information. Because no attempt is made to reject the $\pi^{0}$ backgrounds, the analysis of neutral particle activity remains inclusive of all hadronic final states.

The small "other" category originates with $\pi^{+}$and kaons, mostly from DIS processes which feed down into the sample from higher $q_{3}$ reactions. This category also includes photons and electrons from $\eta^{0}$ production and $\Delta \rightarrow N \gamma$ decay. Photons from GENIE's deexcitation of residual nuclei would be present and isotropic in the data sample but are only simulated for oxygen, not carbon nor other nuclei. Radiative processes from the lepton exiting the nucleus might be collinear with the muon and are also not simulated in GENIE.

The backgrounds from the muon and accidentals are small. The effectiveness of the cuts can be illustrated quantitatively, relative to the base selection. Including the very ends of the detector doubles the accidental background. Not excluding the 17 to $24 \mathrm{~cm}$ outer volume around the muon doubles the muon background. Using an incident neutrino energy range up to $20 \mathrm{GeV}$ increases the statistics of the total data and simulated sample by $17 \%$ and also all the simulated signal and irreducible background subcomponents. Compared to the base selection, these higher-energy reactions have $30 \%$ more in the other category from feed-down of higher $q_{3}$ DIS events. The muon component (because bremsstrahlung is more likely) is also higher by $22 \%$.

Three other cuts could be relaxed to extend the physics reach, but doing so would increase the backgrounds. These samples are consistent with the main sample but do not further enhance the conclusions. Reducing the energy threshold for candidates from 1.5 down to $1.2 \mathrm{MeV}$ increases the predicted rate by $10 \%$ overall, but the data rate increases only by $8 \%$. The predicted muon and accidental background rate increase by $30 \%$ and $50 \%$, respectively, collectively accounting for $20 \%$ of the additional candidates. Allowing the timing to go out to $100 \mathrm{~ns}$ adds events that are predicted to be half from the accidental background.

\section{Efficiency}

Focusing again on the most interesting signal process, the probability that a neutron from the GENIE model produces a cluster of activity is high because of the large volume of the fully active scintillator. The probability to survive the selection rises with kinetic energy from few to $60 \%$. This is shown in Fig. 3, which also illustrates the predicted neutron kinetic energy spectrum for this antineutrino $q_{3}<0.8 \mathrm{GeV} / \mathrm{c}$ sample. Details of the selection process sculpt the distribution. The region near the interaction point especially is a place where $45 \%$ of neutrons leave activity, as one would expect from a mean-free-path process. Some lower-energy neutrons are effectively below

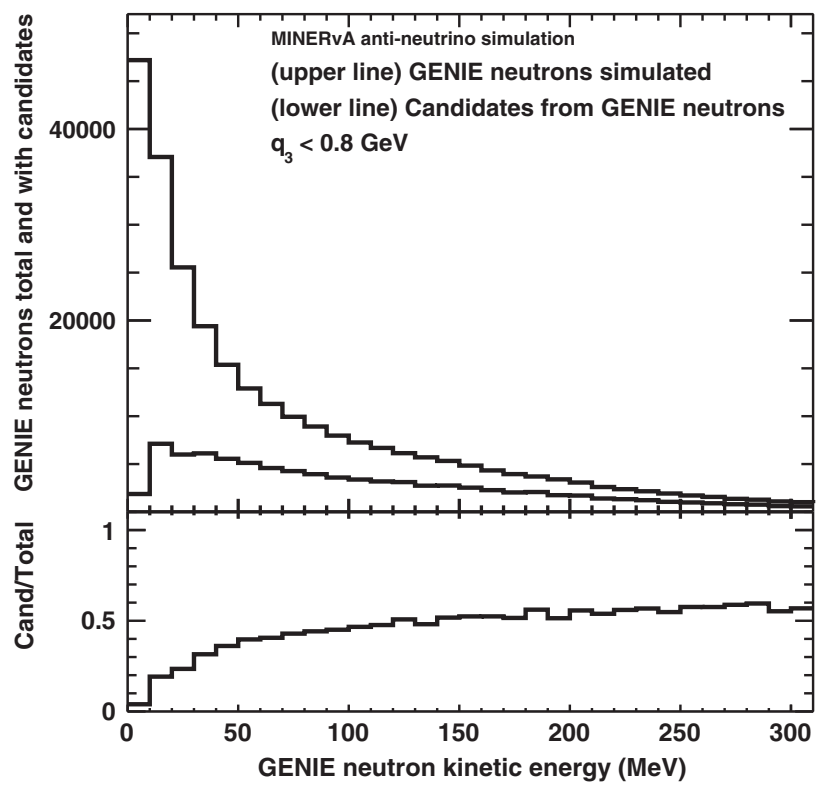

FIG. 3. Distribution of neutrons produced by the GENIE model for the selected $q_{3}<0.8 \mathrm{GeV} / \mathrm{c}$ sample and the subset of neutrons that produced one or more neutron candidates. The ratio in the bottom panel is the efficiency to find at least one candidate from each neutron. 
threshold after just one interaction, and the lowest-energy neutrons do not travel very far and thermalize locally. Higher-energy neutrons may still interact again and be visible.

The efficiency for detecting neutrons using plastic scintillator has been studied since the late 1950s. The 1962 measurement [98] in a scintillator $15 \mathrm{~cm}$ thick with a simple discriminator-based $1.5 \mathrm{MeV}$ electron-equivalent threshold is very similar to the conditions of this MINERvA measurement. They observed an efficiency of $20 \%$ at $76 \mathrm{MeV}$ and $30 \%$ at $10 \mathrm{MeV}$. Below this, the threshold reduces the efficiency significantly. These data have been compared to increasingly sophisticated simulations over the years, such as in Ref. [99]. GEANT4 produces a similar efficiency for neutrons above $20 \mathrm{MeV}$ but predicts closer to $40 \%$ efficiency at $10 \mathrm{MeV}$.

The mix of hydrogen and carbon in the detector affects the efficiency for different ranges of neutron kinetic energy. A special purpose GEANT4 neutron simulation in the MINERvA detector with the same reconstruction as this analysis predicts that hydrogen produces more candidates per nucleus up to neutron kinetic energy of $20 \mathrm{MeV}$ where it is equally probable as detectable scatters from carbon. The hydrogen-to-carbon ratio remains around 1:6 up to $100 \mathrm{MeV}$, then falls further past 1:12 at higher neutron kinetic energies. What is called neutron inelastic scattering in carbon begins around $10 \mathrm{MeV}$ of neutron kinetic energy. (This includes single nucleon knockout, which is the strong-interaction analog to the processes called quasielastic electron scattering and neutral current elastic neutrino scattering.) At lower kinetic energy, neutrons are not able to transfer enough energy to a proton to remove it from the nucleus, yet elastic scattering off hydrogen can produce a proton above the detection threshold. A liquid argon detector would have significantly less acceptance below $20 \mathrm{MeV}$ kinetic energy for the same threshold; a liquid scintillator $\left(\mathrm{CH}_{2}\right)$ detector could have more.

Identifying the presence of a neutron is easier than guessing what the energy of the neutron was. The spectra of energy deposits for neutrons in three different kinetic energy ranges are shown in Fig. 4 (the same figure appears in Ref. [8]). The reconstructed energy of a single cluster or the sum of two or more clusters aggregated into a single candidate is mostly uncorrelated with the kinetic energy of the neutron. The most likely neutron candidate for all kinetic energies considered in this sample is just at the 1.5 MeV threshold.

As the neutron energy rises, the probability it will produce an energetic proton that travels several planes and leaves tens to hundreds of mega-electron-volts also rises and populates the distribution at and beyond the right edge of the plot up to hundreds of mega-electron-volts.

The converse is also relevant. Neutrons in the range 10 to $20 \mathrm{MeV}$ can only make the smallest energy deposits. The presence of a small energy deposit does not automatically

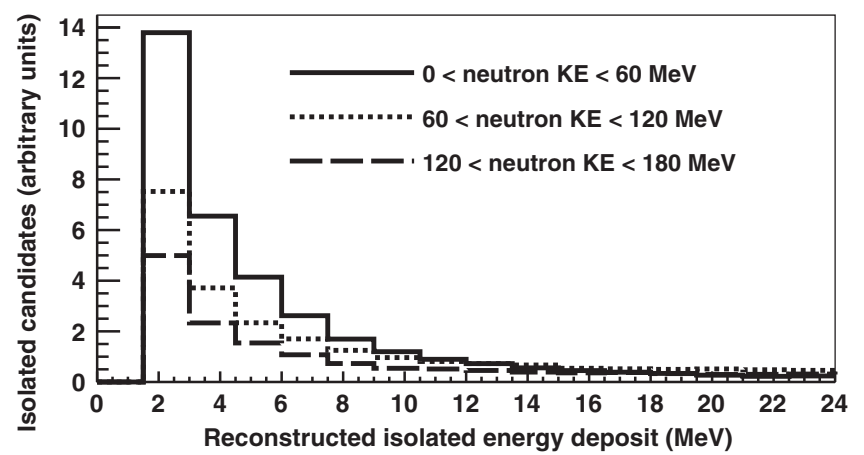

FIG. 4. Predicted candidate energy spectrum from GENIE neutrons in three energy ranges from the selected sample. The similarity of the spectra prevent a robust, direct calorimetric neutron energy measurement.

indicate a low-energy neutron. But the presence of many low-energy neutrons can only enhance the rate of the smallest energy deposits.

Overall, the presence of neutrons down to $50 \mathrm{MeV}$ kinetic energy is determined with good efficiency and low backgrounds. Though the efficiency continues to fall, neutrons down to $10 \mathrm{MeV}$ are predicted to cause a substantial part of the sample.

\section{MEASUREMENTS OF NEUTRON ACTIVITY}

One observation already stands out from Table II. There is an overall overprediction of neutron candidates in the simulation, further emphasized in Table III. It appears in all subdivisions of the sample, despite different amounts of $\mathrm{QE}, 2 p 2 h$, and $\Delta$ resonance and their varying neutron finalstate content. Because the efficiency is so high and the backgrounds are so low, either GENIE is producing too many neutrons per event or the GEANT4 neutron propagation plus detector response simulation is making them more visible than in the data. Compared to Table III, before modifying the GEANT4 cross section to match the Abfalterer et al. measurements, the neutron candidates per event were 1.20 for each range of momentum transfer.

In this section, distributions of deposited energy, time, and position upstream or downstream are shown. Then, neutron speed (actually $1 / \beta$ ) and multiplicity per event are

TABLE III. Ratio of simulation to data for selected events, neutron candidates, and candidates per event. All systematic uncertainties are accounted for except for two model variations treated separately and described later.

\begin{tabular}{lcc}
\hline \hline & \multicolumn{2}{c}{3 -momentum transfer } \\
\cline { 2 - 3 } MC/data & $0<q_{3}<0.4$ & $0.4<q_{3}<0.8$ \\
\hline Selected events & 0.92 & 1.03 \\
Neutron candidates & 1.02 & 1.20 \\
Neutron candidates/event & 1.11 & 1.16 \\
Statistical uncertainty & 0.02 & 0.01 \\
Systematic uncertainty & 0.07 & 0.04 \\
\hline \hline
\end{tabular}


used to draw final conclusions. Starting with the time distributions, the low-energy candidates and high-energy candidates are separated, and the oversimulation persists in the former.

In all cases, the reconstructed data distribution is shown with statistical uncertainties only (often too small to see), and the simulation is shown with model and detector systematic uncertainties. Some physics effects being tested with these data are not included in the systematic uncertainties and are described explicitly. Each figure is neutron candidates per event like Table III, reducing systematics that affect the numerator and denominator equally, such as the flux and some cross section uncertainties. Discussion of specific uncertainties and resolutions are provided with each new distribution, if not previously described.

In each plot, the simulation is broken down into GENIE neutrons, other sources of neutrons, and the electromagnetic component. The muon and accidental backgrounds are often too small to see and are described in the text instead of being plotted. Each figure has a lower, ratio subpanel where the data-to-simulation ratio is compared to the reference model and its uncertainties, to emphasize the magnitude and location of discrepancies.

\section{A. Spectrum of candidate deposited energy}

The energy deposit spectrum $E_{\text {dep }}$ shown in Fig. 5 highlights that the extra neutron candidates in the simulation are limited to those with less than $10 \mathrm{MeV}$. They are unambiguously from neutron production. The predicted neutron components of the spectrum are much higher and peak much more strongly at threshold than the electromagnetic component, shown by the dotted line in Fig. 5. The estimate of the backgrounds is not subtracted and is a $12 \pm 1 \%$ contribution to the first bin in each histogram (not shown with its own line but similar to the proton + pion population in those two bins) and negligible everywhere else. Above $10 \mathrm{MeV}$, the data are described by the

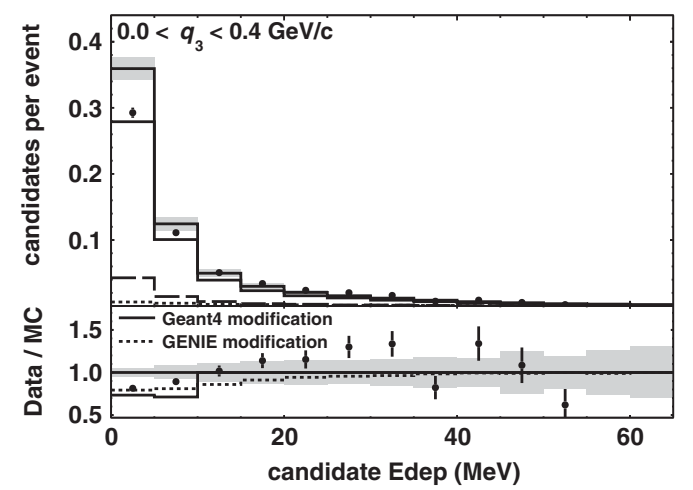

simulation, allowing for the systematic uncertainties summarized by the shaded region.

We use these data to probe for model features beyond the standard systematic uncertainties included in the error band. The ratio subpanel includes two modified models we will refer to as "benchmarks" throughout this section. For the solid "modified GEANT4" line, we eliminate a random 50\% of neutron candidates with less than $10 \mathrm{MeV}$ energy deposit and originating from neutron-producing GENIE particles (neutrons, protons, $\pi^{ \pm}$). Without regard to the energy of the particle, this mimics moving part of the GEANT4 or detector response below our detection threshold, making these elastic scatters invisible or reducing the number of photons produced by nuclear deexcitations in carbon following the nucleon knockout process. The dotted line implements a neutrino-interaction model change; $50 \%$ of candidates caused by GENIE neutrons below kinetic energy of $50 \mathrm{MeV}$ are removed. This makes the prediction more like NuWRO in Fig. 1. These benchmark modifications are chosen empirically to have about the right size and allow the analysis to track their effects across the rest of the distributions.

Considering Fig. 4, neutron-caused candidates above $10 \mathrm{MeV}$ are necessarily from higher kinetic energy neutrons, while the lowest-energy candidates are a mix of everything. By itself, the modified GENIE benchmark that reduces only the lowest-energy neutrons would provide a description of the $0.0<q_{3}<0.4 \mathrm{GeV} / \mathrm{c}$ region at the edge of the systematic error band. But its effects are not strong enough to describe the right panel. The GEANT4 benchmark that removes only low-energy candidates would describe both regions adequately, and the 5 to $10 \mathrm{MeV}$ point in the left plot would remain slightly outside the error band.

\section{Uncertainties}

Signal response uncertainties that affect the probability a neutron will produce a cluster near the $1.5 \mathrm{MeV}$ threshold are important. Neutron elastic scatters produce a low-energy

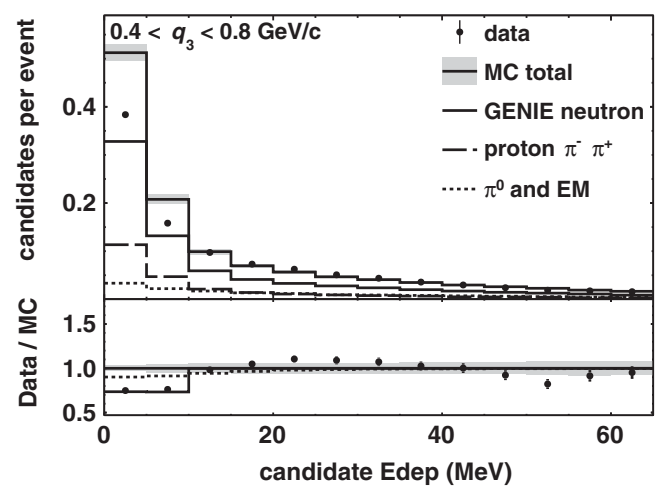

FIG. 5. Distribution of measured energy deposit $E_{\text {dep }}$ per neutron candidate, normalized by the total number of events. Data are shown with statistical uncertainties only; the simulation is shown with systematic uncertainties. The lowest bin only contains candidates down to the $1.5 \mathrm{MeV}$ threshold. The lower panels contain ratios to the reference simulation for the data and for modifications to the GENIE and GEANT4 simulations, which will serve as a benchmark and are described in the text and used hereafter. Bins with very large data statistical uncertainties are not shown. 
proton of which the detector response is affected by a scintillator quenching effect parametrized using Birks's Law $[100,101]$. Our parameter and its uncertainties are calibrated using test beam data from stopping protons [14] especially the last $40 \mathrm{MeV}$ of their energy deposits. The uncertainty is doubled for neutron-induced candidates with less than $5 \mathrm{MeV}$, which are not well constrained by the test beam data. The total Birks' uncertainty changes the response as much as $25 \%$ for candidates near threshold. Increasing the Birks suppression migrates events down in these distributions, and some fall below the $1.5 \mathrm{MeV}$ threshold. Modern studies of Birks's quenching in liquid and plastic scintillators [102,103], made for supernova and solar neutrino detection, are also at these energies and confirm the predicted scintillator response. This uncertainty is the largest single contribution in the first bin of Fig. 5 but is only $4 \%$.

The GEANT 4 cross section model uncertainties play a lesser role in this distribution than they do in the time and spatial distributions later. An increase in the cross section (decrease in the mean free path) makes candidates interact earlier. Candidates are more likely in and near the interaction exclusion region and there are fewer candidates overall. It contributes $4 \%$ to the uncertainty but only $2 \%$ in the first bin. The GEANT4 energy deposit model and photon yield are further explored using the benchmark modification shown with the solid black line in the ratio panels.

Large rate uncertainties on the QE, $2 p 2 h$, and resonance models combine for $3 \%$ uncertainty on this distribution, but
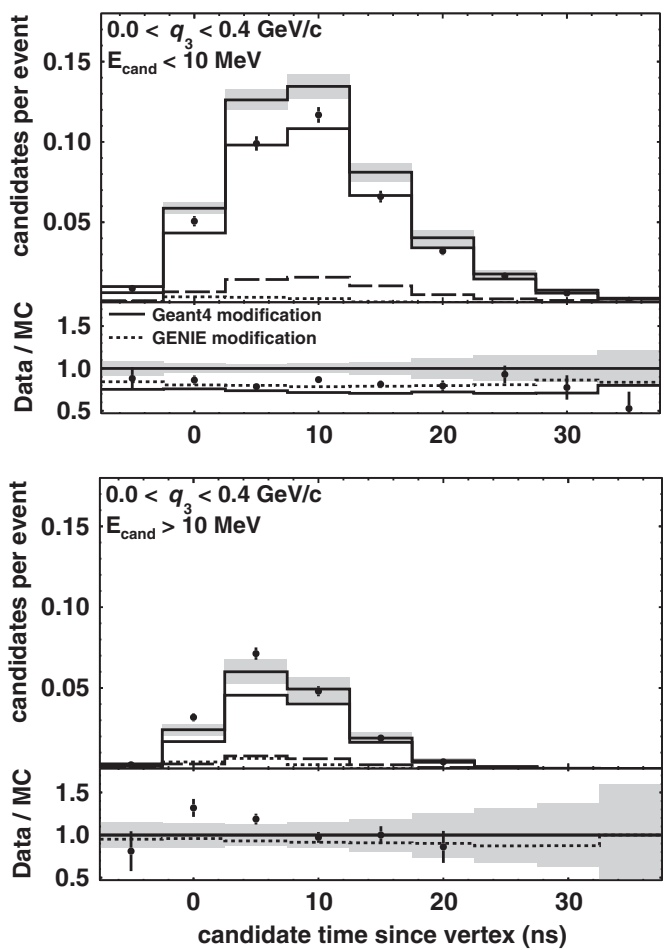

less than $1 \%$ uncertainty in the first two bins, and are the largest source for most bins for $0.4<q_{3}<0.8 \mathrm{GeV} / \mathrm{c}$. All these antineutrino processes produce some neutrons with similar energies. Distortions of the energy transfer spectrum for these processes, such as the uncertainty assigned to the RPA screening effect for QE used in the reference model [51,53], are at 4\% and only important for the $q_{3}<$ $0.4 \mathrm{GeV} / \mathrm{c}$ subsample beyond the first two bins.

Effects related to the hadronic energy scale act in a special way and are significant for energy deposits above $10 \mathrm{MeV}$. They cause a migration of events up and down the range of $q_{3}$. This migration effect is most significant and dominates parts of the error band for $q_{3}<0.4$. The subsample is lower statistics, has fewer candidates per event overall, and does not have compensating event feedin/-out at its lower boundary. The hadronic energy scale uncertainties are assigned based on test beam data with an enhanced uncertainty for the neutron response that was not directly tested. The GENIE FSI uncertainties also play this same migration role, in addition to directly changing the number of candidates in each event, and contribute a similar amount to total uncertainty.

\section{B. Time-of-flight distribution}

The time difference between the neutron candidate and the interaction time, $t_{n}-t_{0}$, shown in Fig. 6 produces separation of the prompt electromagnetic component from
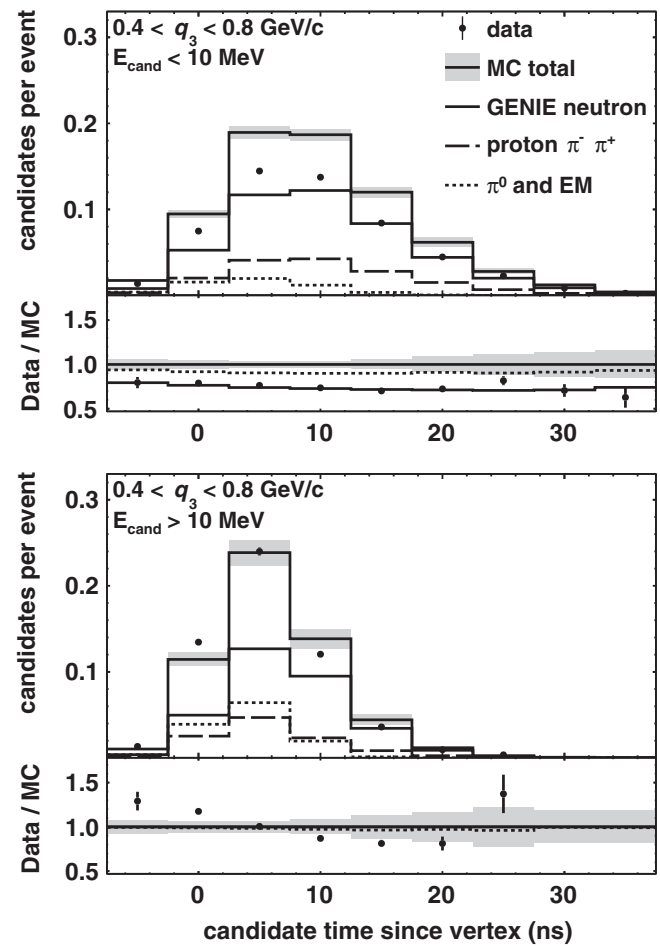

FIG. 6. Time of the candidate relative to the time of the interaction. Data are shown with statistical uncertainties only; the simulation is shown with systematic uncertainties. Neutron candidates with energy deposits less than $10 \mathrm{MeV}$ are shown for both ranges of $q_{3}$ in the upper plots, and higher-energy candidates are the lower plots. Bins with very large data statistical uncertainties are not shown. 
the slowest neutron component. Because the simulated overprediction is so prominent in the first two bins, the following distributions will be separated into subsamples below and above $10 \mathrm{MeV}$ of candidate energy deposit $E_{\text {dep }}$. The simulation's overprediction for candidates with reconstructed energy less than $10 \mathrm{MeV}$ in the upper panels appears roughly uniform across all high-statistics bins of the time of flight. For higher-energy candidates in the lower panels, there are trends beyond the edge of the error band to relatively overpredict the latest times, from neutrons that traveled either the farthest or the slowest. The modified GEANT4 response model describes the data better than the modified GENIE model for the $0.4<q_{3}<0.8 \mathrm{GeV} / \mathrm{c}$ topright panel. By construction the GEANT4 benchmark has no effect on the lower panels, though the GEANT4 cross section uncertainties are significant in the error band. The GENIE modification reduces the slowest neutrons in the sample and has a slight shape effect smaller than the predicted error band.

Fluctuations to negative times are compatible with the time resolution of $4.5 \mathrm{~ns}$ for single-cluster candidates (shown later in Fig. 9): about one bin in these histograms. Systematic uncertainties directly from the measurement of time of flight for an individual event contribute negligibly. The simulation of the timing distribution is taken from a separate, in situ muon sample. The lack of bias is independently confirmed using clusters on the muon tracks
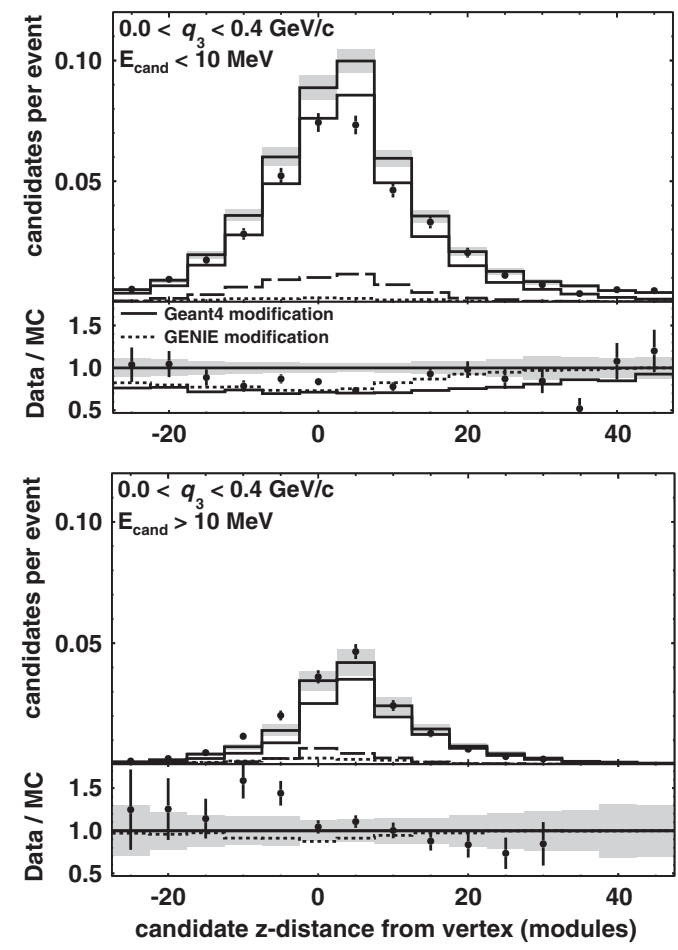

of interactions in the selected sample and reconstructed the same as neutron clusters.

The same systematics described in the previous section are evaluated for this distribution. The GEANT4 neutron cross section model uncertainties enhance or reduce the appearance of neutrons that travel the farthest and so have the longest times. It dominates the error bar in all bins beyond $15 \mathrm{~ns}$. The other uncertainties described previously contribute roughly equal amounts in the center of the distribution.

\section{Position upstream or downstream}

The overprediction of candidates with energy deposits less than $10 \mathrm{MeV}$ appear broadly around the interaction point, shown in Fig. 7. In the $E_{\text {dep }}<10 \mathrm{MeV}$ samples, the oversimulation may be prominent near the interaction point in the top left plot, while it spans the detector for the higher momentum transfer sample in the top-right plot. For higherenergy candidates, the simulation does well overall except for two underpredicted bins in the backward direction of the $q_{3}<0.4 \mathrm{GeV} / \mathrm{c}$ panel (lower left).

There are more neutron candidates in the forward direction, where the $\mathrm{QE}$ process is especially relevant. In contrast, candidates from any process involving multiple particles can end up going backward from the interaction point. This includes multibody reactions $2 p 2 h, \Delta$, and FSI
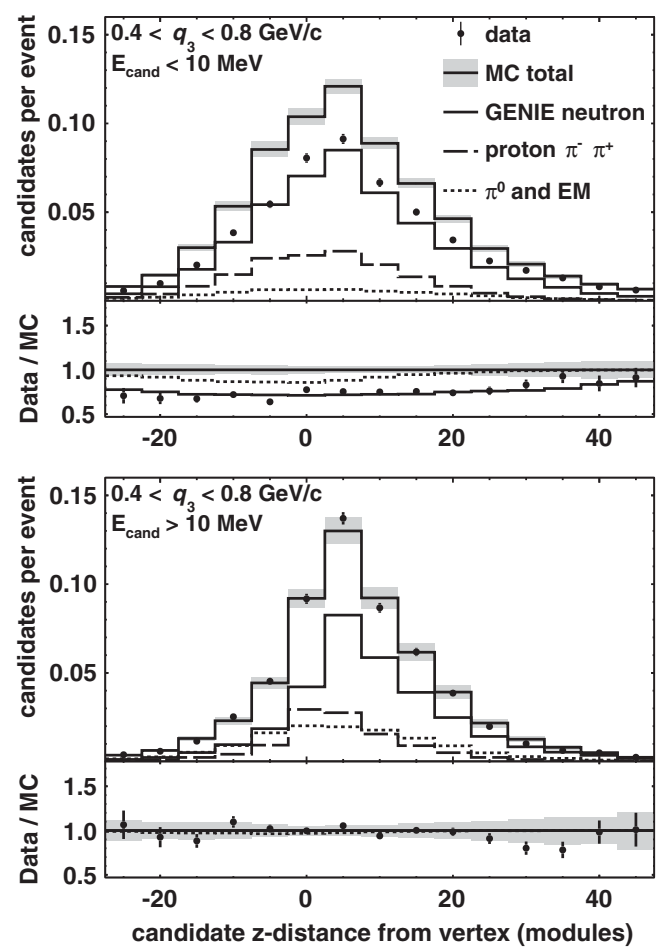

FIG. 7. Position of the candidate relative to the interaction point in the upstream/downstream direction. Data are shown with statistical uncertainties only; the simulation is shown with systematic uncertainties. Neutron candidates with energy deposits less that $10 \mathrm{MeV}$ are shown for both ranges of $q_{3}$ in the upper plots, and higher-energy candidates are the lower plots. Bins with very large data statistical uncertainties are not shown. 
in the nucleus and neutrons produced when protons and pions and neutrons reinteract in the detector.

The cross section for neutron reinteractions is the direct and dominant uncertainty in the downstream region, as it was for long times. Again, the other uncertainties contribute roughly equally in the peak of the distribution.

\section{Particle speed}

We estimate the apparent speed of the particle as a fraction of the speed of light, $\beta$. Because the timing resolution plays a crucial role, and for better treatment of zero and negative times, it is more clear to present

apparent $1 / \beta=$ speed of light $\times$ time $/ 2 \mathrm{D}$ distance.

The result is shown in Fig. 8.

The estimate of a two-dimensional (2D) distance can be made of the hypotenuse of the distance in $\mathrm{Z}$ shown Fig. 7 and the distance along the one $\mathrm{X}, \mathrm{U}$, or $\mathrm{V}$ transverse direction measured for single-cluster candidates. This distance is transverse from the neutrino's path, not from the muon. If the neutron candidate is made of activity in more than one plane, the longest transverse position is used. This distance estimator is necessarily smaller than the true distance the neutron traveled because it is missing the third of three coordinates and because some neutrons bounce and take an indirect path to the point where an energy deposit is
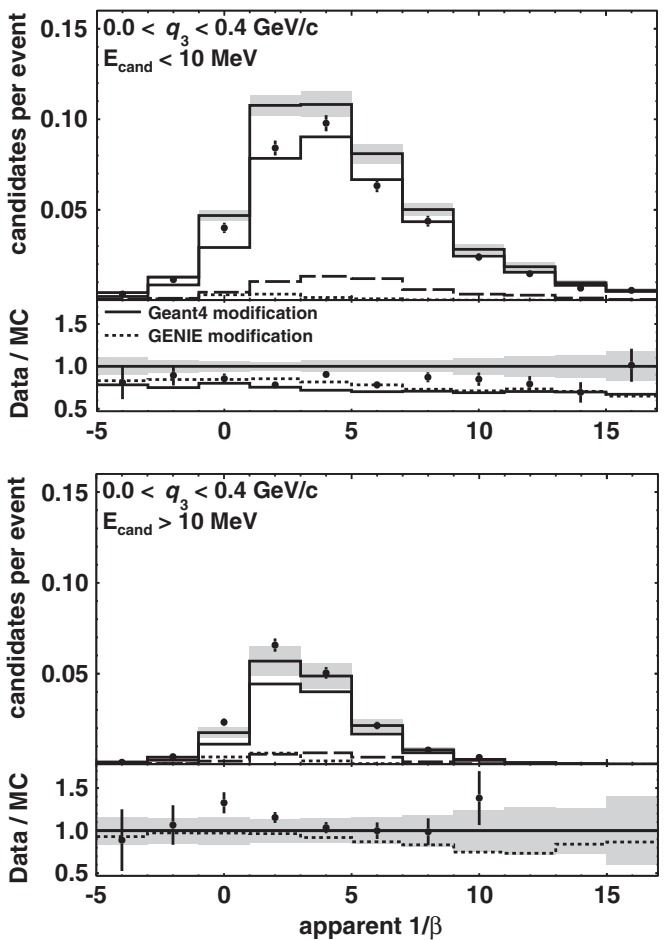

observed. This distribution has properties similar to the one in Fig. 7 and is not shown.

The systematic underestimate of the $2 \mathrm{D}$ distance means a systematic overestimate of $1 / \beta$ of about 0.8 and a rms resolution between 2 and 3, driven largely by the timing resolution. The resolution for the slowest particles with true $1 / \beta>5$ is the worst because they do not travel very far and are observed closest to the interaction point. They have a resolution of around 4 and a bias of -0.8 . The detector-only (without the effect of neutron multiple scatter) time and 2D distance resolutions are shown in Fig. 9. For neutrons, $1 / \beta=5$ implies $20 \mathrm{MeV}$, and $1 / \beta=10$ implies $5 \mathrm{MeV}$; however, the latter are expected to rarely produce candidates (see again Fig. 3), and the population beyond $1 / \beta=$ 10 must be from fluctuations in the assigned time and distance. The resolutions and thresholds are such that the apparent $1 / \beta$ is not usefully transformed into a kinetic energy distribution.

The GEANT4 cross section uncertainty, prominent in the time and $\mathrm{z}$ distance distributions separately, is much reduced and has little shape. A smaller or larger mean free path in the simulation affects both the time and the distance simultaneously. Other uncertainties contribute similarly across these distributions. The hadronic energy scale and FSI uncertainties (migrations in $q_{3}$ ) are especially significant in the first four bins of both the $E_{\text {cand }}>10 \mathrm{MeV}$ (lower plots), which is where discrepancies remain.

The electromagnetic component peaks near $1 / \beta=1.8$, shown as the dotted line in the upper panels of each figure.
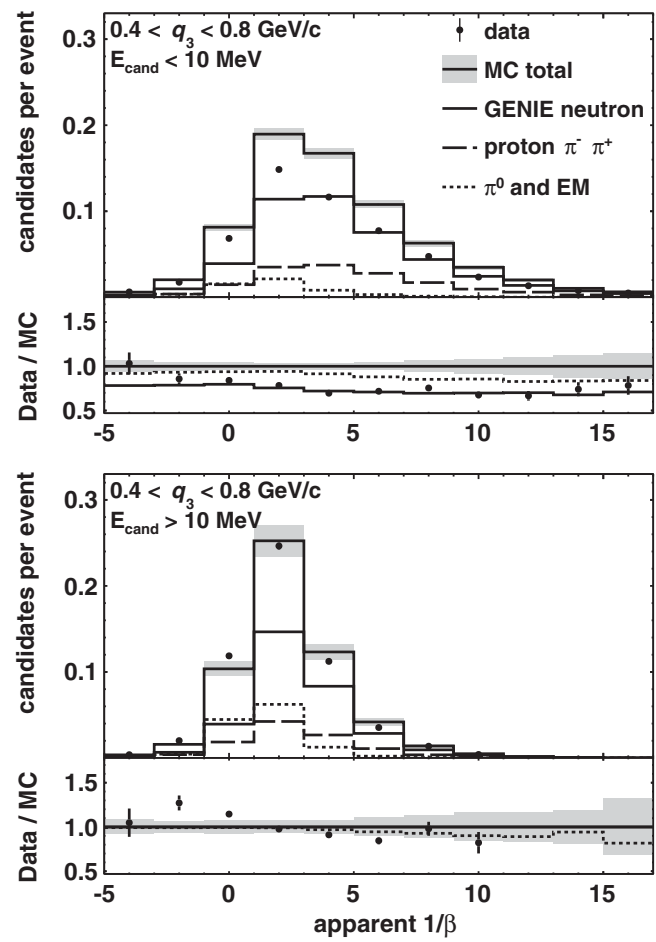

FIG. 8. Apparent $1 / \beta$ of the particle causing the neutron candidates, expressed as a fraction of the speed of light. Data are shown with statistical uncertainties only; the simulation is shown with systematic uncertainties. Bins with very large data statistical uncertainties are not shown. 

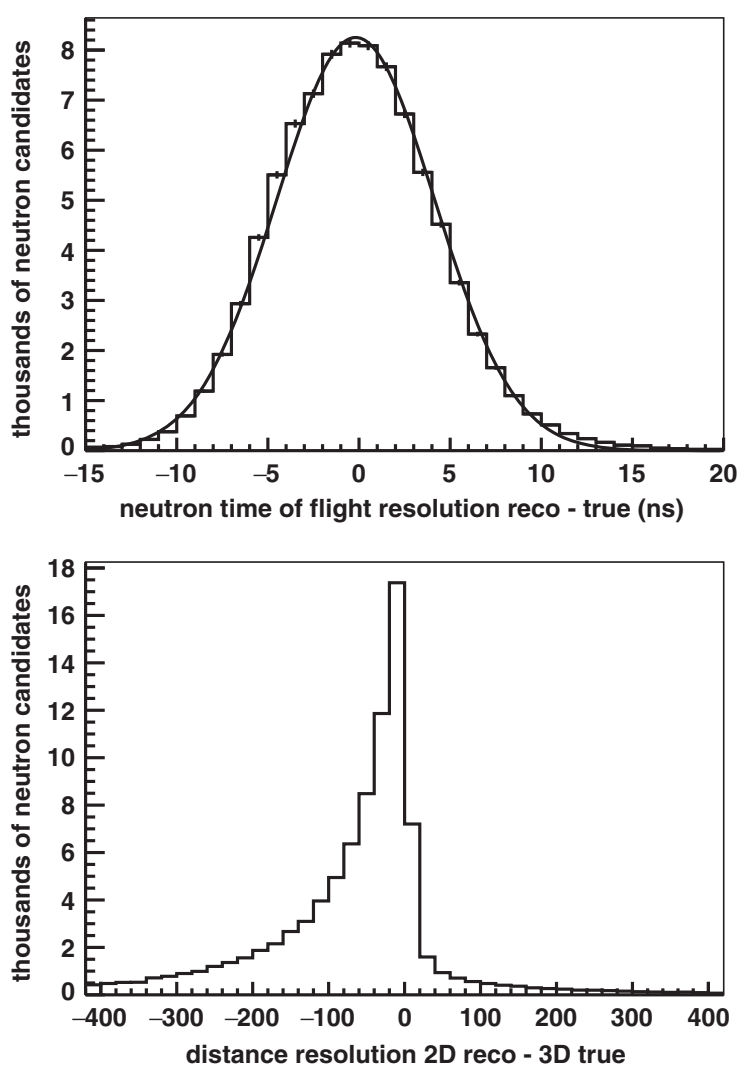

FIG. 9. Detector resolutions on the time and distance inputs to $1 / \beta$ from the simulation, only for neutron candidates of which the origin was a neutron from the GENIE simulation. There is no timing bias, and the Gaussian fit to the timing resolution has $\sigma=4.5 \mathrm{~ns}$. At the speed of light, the mean and $\mathrm{rms}$ of the distance distribution correspond to 0.15 and $0.3 \mathrm{~ns}$. Neutron multiple scattering effects are not included in the lower plot.

The neutron component peaks instead near $1 / \beta \sim 4$ for candidates from the lowest kinetic energy neutrons and $1 / \beta \sim 2.5$ for faster neutrons. The GENIE benchmark modification produces a reduction in the slowest neutron component that goes in the direction of the data but again does not match the overall magnitude of the discrepancy in the upper right panel.

\section{E. Neutron multiplicity}

The overprediction of neutron candidates per event in the simulation also distorts the multiplicity of candidates per event, shown as a percent of the total in Fig. 10. The difference in percent (not the ratio) between the data and the reference simulation is shown in the first panel below the main distributions for compact comparison. The overprediction of neutrons masks the presence of the $2 p 2 h$ component and RPA screening because both also enhance the percent of events with neutron candidates. Again, the GENIE and GEANT4 serve as benchmark modifications showing the (modified simulation - reference) in percent in the lower two rows, but now to expose these multinucleon features of the data. The two regions of $q_{3}$ shown in the previous figures are subdivided according to hadronic energy to produce distributions for QE-rich, dip, and $\Delta$-rich subsamples, six in total. In the top panels, the oversimulation of neutron candidates is most evident in the dip and $\Delta$-rich subsamples where the simulation significantly overpredicts how many events have three or more neutron candidates and underpredicts how many have none.

The QE-rich subsets uniquely offer predicted sensitivity to multinucleon effects. The long dashed line in Fig. 10 completely removes the simulated $2 p 2 h$ component. These events preferentially had multiple neutrons in the first place, so removing them increases the bin with zero candidates. The short-dashed line shows that removing also the QE RPA screening effect adds back events of which the outgoing neutron was lower energy and less likely to make a candidate, also increasing the bin with zero candidates. In contrast to the QE-rich panels, there is no sensitivity to these multinucleon effects in the other panels; all reactions produce similar numbers of neutrons after FSI. Variations of RPA and $2 p 2 h$ processes are the same size as the uncertainty bands in those panels.

A different way to summarize the subdivision of the data: neutrino model details in Fig. 10 are orthogonal to the neutron details in the previous figures. Modifications to the $\mathrm{QE}$ and $2 p 2 h$ models show up in QE-rich region here, while the excess of neutrons distorts all six panels similarly. The opposite happens in the previous figures; distortions of the spectra due to neutron production details are evident, but modifications to the $2 p 2 h$ and QE models are largely flat with neutron candidate time, position, and speed.

What is desirable is to tune the neutron model to the dip and $\Delta$-rich regions, a common technique when there are sidebands to a signal selection. Such a tune would correct and constrain the mismodeled neutron effects in the multinucleon sensitive distributions. Though we do not directly have tunable parameters, a simplified version is obtained by remaking the distributions while applying the benchmark modifications to GEANT4 (third row) and GENIE (bottom row). The resulting dip and $\Delta$-rich regions are now consistent with uncertainties for $0<q_{3}<0.4 \mathrm{GeV} / \mathrm{c}$. The GEANT4 modification produces better distributions for $0.4<q_{3}<0.8 \mathrm{GeV} / \mathrm{c}$, perhaps overcorrecting, while the GENIE modification produces mild improvement that does not go far enough. Both roughly mimic the behavior of these benchmarks in the energy, time, position, and speed distributions.

The benchmark modifications also reduce the preponderance of simulated neutron candidates in the QE-rich signal region. This enables further interpretation of the presence of multinucleon effects and other unsimulated processes. Especially in the leftmost two bins in the lower two rows of panels, the modified simulations now have a $6 \%$ to $8 \%$ underprediction of events with one neutron 

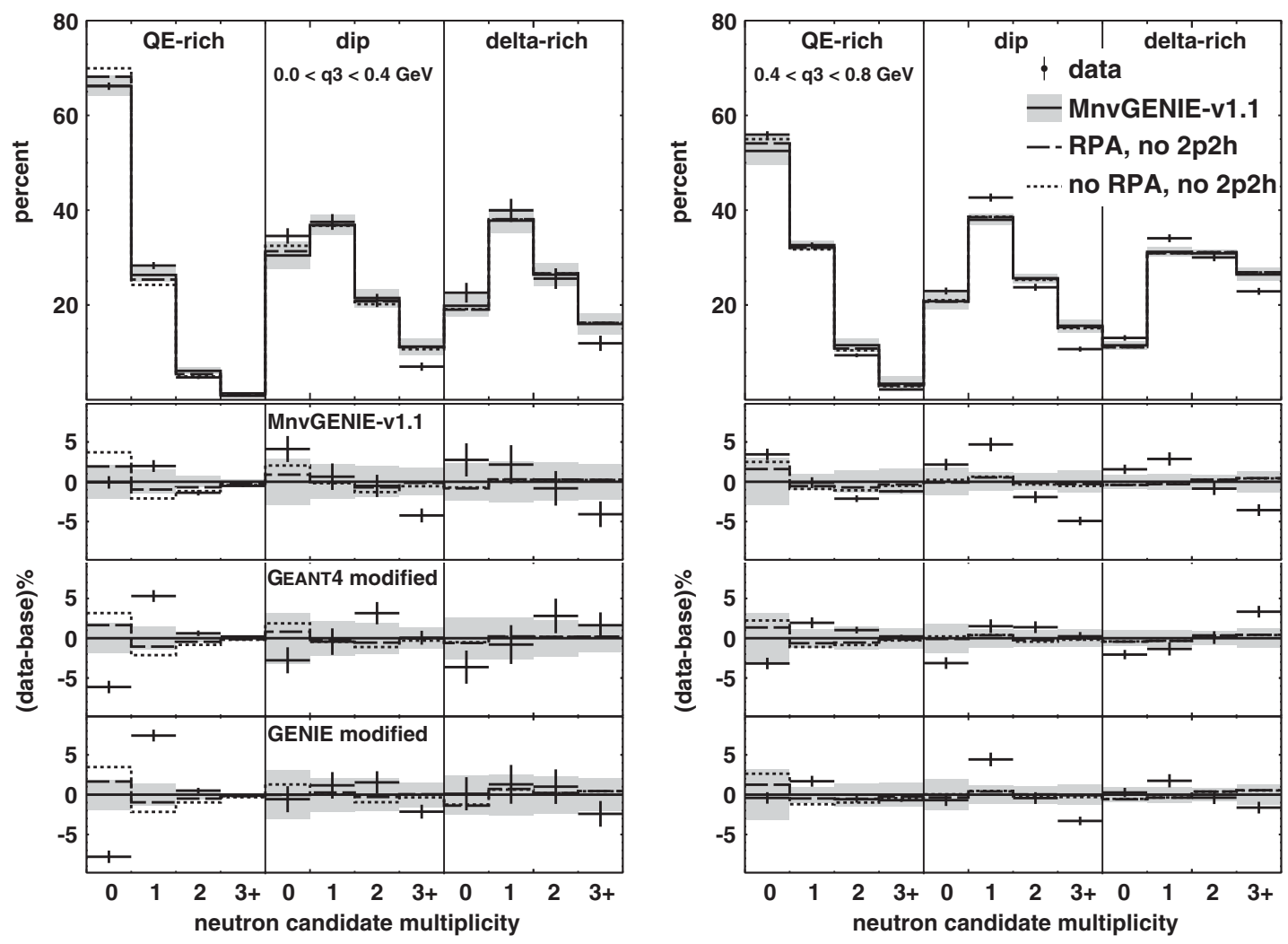

FIG. 10. Candidate multiplicity distribution for all six subsamples, $0<q_{3}<0.4$ (left) and $0.4<q_{3}<0.8 \mathrm{GeV} / \mathrm{c}$ (right), with subpanels for the QE-rich, dip, and $\Delta$-rich regions. The top plot shows the reference MNVGENIE-V1.1 simulation with a solid line and error band and two variations that turn off completely the $2 p 2 h$ component and then also turn off the RPA component. The next row shows the difference from the reference simulation. The middle (lower) row of difference plots uses the modified GEANT4 benchmark (modified GENIE benchmark) for all distributions.

candidate and an overprediction of events with none. This is roughly two times the combined effects of our current RPA, $2 p 2 h$ multinucleon models and all systematic uncertainties. In both cases, the resulting new predictions hint the data want even more $2 p 2 h$ interactions or RPA screening than the reference MNVGENIE-v1.1 simulation.

Sideband tuning usually takes a poor model and improves it before extracting the physics quantities of primary interest. In this case, it takes what was naively a reasonable description in the QE-rich sample and indicates disagreement beyond the available multinucleon effect models. However, all the relative trends (not shown) of the whole sample in energy, distance, time, and speed shown in previous figures are also present for the QE-rich sample, suggesting that the sideband adjustment will succeed and the new disagreement is a robust observation. Alternatives to simply adding more $2 p 2 h$ or RPA screening that would correct the resulting model disagreement are to add an additional process like deexcitation photons from carbon or a more nuanced, QE-specific version of either of the benchmark modifications.

In all the previous distributions, the current error bands preclude further detailed tests of the magnitude of RPA, the relative proton + neutron and neutron + neutron content of the $2 p 2 h$ process, and the need for a low $Q^{2}$ suppression of resonances [104]. The sensitivity would be limited even if there were no large discrepancy, but modified RPA, $2 p 2 h$, or resonances would not explain the $E_{\text {dep }}<10$ in the whole sample nor in the QE-rich sample on its own.

Prior MINERvA measurements show distributions with sensitivity to the RPA and $2 p 2 h$ multinucleon models that may also be sensitive to neutron effects. In Fig. 3 of Ref. [8], the reconstructed hadronic energy for this same sample is improved with the addition of RPA and a tuning of $2 p 2 h$ to the neutrino data in Ref. [9], but the antineutrino agreement is not perfect. The untracked energy within $100 \mathrm{~mm}$ from the interaction point of antineutrino reactions in Fig. 25 of Ref, [68] is effectively in the excluded region of this analysis. That distribution is also not as well described by MNVGENIE-v1 compared to the equivalent Figs. 35-36 of Ref. [70]. In both examples, and based on the neutron observations in this paper, the description of the antineutrino data could be improved with a reduction of the neutron component of the reconstructed energy in the simulation, while having a smaller effect on the neutrinomode data. Such a mechanism supposes the reduced neutron energy goes missing rather than being offset by additional charged hadron energy. 

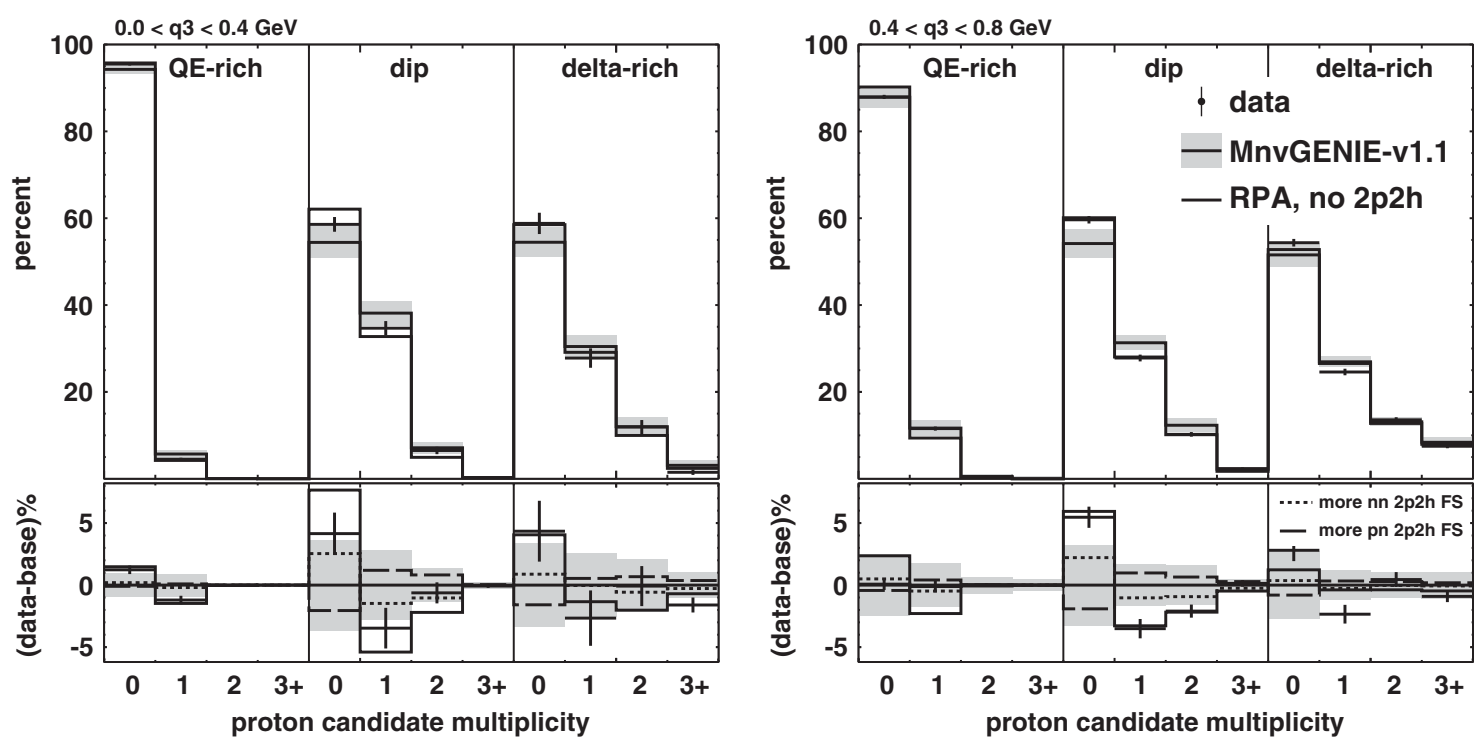

FIG. 11. Candidate proton multiplicity distribution for all six subsamples, $0<q_{3}<0.4$ (left) and $0.4<q_{3}<0.8 \mathrm{GeV} / \mathrm{c}$ (right), with subpanels for the QE-rich, dip, and $\Delta$-rich regions. The top plot shows the reference MNVGENIE-V1.1 simulation with a solid line and error band and one variation that turns off completely the $2 p 2 h$ component. The subpanels show the difference from the reference MNVGENIE-V1.1 for two additional $2 p 2 h$ variations: the dotted line enhances only the $p n$ initial states which give $n n$ final states (FS), and the dashed line enhances only the $p p$ initial states leading to $p n$ final states.

\section{F. Protons}

This final study is the analog to the untracked protons reported for the related neutrino interaction sample of Ref. [9], where the proton multiplicity with an untuned Valencia $2 p 2 h$ process is at the edge of the error band of a prediction without it. Protons are counted by observing single strips with at least $20 \mathrm{MeV}$ near the interaction point, indicating the Bragg peak at the end of the proton range. Because the single strip could be at the interaction point itself, the threshold is effectively just above $20 \mathrm{MeV}$. T2K has recently presented results for proton multiplicity [105] using a tracking threshold kinetic energy of $100 \mathrm{MeV}$.

Protons are also more common in the final state of a $2 p 2 h$ antineutrino reaction, relative to antineutrino $\mathrm{QE}$ reactions. Repeating that earlier strategy reveals marginal sensitivity, shown in Fig. 11. The reference simulation is shown with a solid line and systematic error band, a simulation with no $2 p 2 h$ process at all is shown with the second solid line that has fewer proton candidates and more zero-proton events. The RPA screening applied to the QE process has a negligible effect on this proton distribution, and an additional line without it is not included. Two additional model variations are shown in the lower panels as the difference from the reference simulation. The dotted line enhances (above the Valencia $2 p 2 h$ model) only the $p n$ initial states which lead to $n n$ final states and predict a higher fraction of events with no protons. The dashed line enhances only the $p p$ initial states, which lead to $p n$ final states and produce more detectable protons.

Given the uncertainties, the proton multiplicity data are consistent with all model variations presented. Visually, the preference is for models that have fewer protons, either from less $2 p 2 h$ overall or from $2 p 2 h$ reactions that favor neutron only final states. The latter is also the conclusion from electron scattering results that indicate that $p n$ shortrange correlated pairs are significantly more common [106] than like-nucleon pairs. The GENIE FSI model uncertainties play the most significant role in degrading the sensitivity because they control how many additional protons are ejected from the nucleus, especially for QE reactions. This sensitivity was not as strong in the neutrino case [9] where outgoing protons are the direct products of the reaction. Both FSI and calibrated hadronic energy scale uncertainties have a significant effect on the QE-rich panel where a higher-energy scale causes a migration of events to the dip region or higher three-momentum transfer panel. Finally, the Birks suppression uncertainty is also significant throughout these distributions; its size is half the total uncertainty shown. It makes simulated protons more or less likely to pass the $20 \mathrm{MeV}$ selection. Because $2 p 2 h$ variations are shown explicitly, no uncertainty on the $2 p 2 h$ process is included in the error band.

Liquid argon experiments [107-110] have shown more low-energy, charged proton tracks in the simulation compared to data. Under ideal circumstances, this detector technology permits tracking of protons with as little kinetic energy as $21 \mathrm{MeV}$. The GENIE model also produces more low-energy protons than other neutrino event generators, correlated with its behavior for neutrons. This supports that the GENIE benchmark modification may be part of resolving these discrepancies. In MINERvA, protons under $20 \mathrm{MeV}$ would not meet the threshold 
for detection. They would usually deposit all their energy in the same scintillator strip in which the reaction occurred, and it takes $100 \mathrm{MeV}$ before protons start to be trackable. So, unlike neutrons and unlike protons in liquid argon detectors, multiple low-energy protons in a single scintillator strip in MINERvA would be counted only once, if at all.

\section{CONCLUSION}

We have obtained the first time of flight, spatial, and speed $(1 / \beta)$ distributions of neutrons from antineutrino interactions. The reference simulation, the components of which are widely used by neutrino oscillation experiments, overestimates the number of neutron candidates by $15 \%$ overall but by $25 \%$ for energy deposits less than $10 \mathrm{MeV}$, shown in the upper figures of Figs. 6-9. A possible interpretation is that the GENIE neutrino event generator and its FSI model are overproducing the lowest-energy neutrons. Also likely, the GEANT4 and detector models turn too many neutron interactions into measurable activity. Combinations and variations of these two benchmark modifications are paths forward. The discrepancy is around two standard deviations from the combination of the other sources of uncertainty.

Additional distortions may be present for candidates with energy deposits more than $10 \mathrm{MeV}$. The MC overestimates long times of flight relative to short in Fig. 6, far and forward relative to near and backward in Fig. 7, and slow relative to prompt in Fig. 8. These discrepancies are just beyond the error band, suggesting one or more of the uncertainties come close to accounting for these data.

It is a reasonable assumption that similar overproduction of small energy deposit neutron candidates is present for all subcomponents of the sample. In this case, the multiplicity distribution in the QE-rich subsamples indicates a preference for a model that has a combination of RPA screening and a $2 p 2 h$ component, both of which reduce the relative proportion of events with zero neutron candidates.

\section{ACKNOWLEDGMENTS}

We are grateful to Juan Nieves, Ignacio Ruiz Simo, and Manuel Vicente Vacas for making their RPA and $2 p 2 h$ code available for study and incorporation into this analysis. Samples of other neutrino event generator neutron predictions were prepared by Jake Calcutt, Luke Pickering, and Kendall Mahn using the NUISANCE framework. This document was prepared by members of the MINERvA Collaboration using the resources of the Fermi National Accelerator Laboratory, a U.S. Department of Energy, Office of Science, HEP User Facility. Fermilab is managed by Fermi Research Alliance, LLC (FRA), acting under Contract No. DE-AC02-07CH11359. These resources included support for the MINERvA construction project, and support for construction also was granted by the United States National Science Foundation under Grant No. PHY0619727 and by the University of Rochester. Support for scientists for this specific publication was granted by the United States National Science Foundation under Grants No. PHY-1306944 and No. PHY-1607381. We are grateful for the United States National Science Foundation's decade of direct support to the Soudan Underground Lab outreach program, including Grant No. PHY-1212342; this analysis originated as the research component for two summer undergraduate outreach interns. Support for participating scientists was provided by NSF and DOE (USA) by CAPES and CNPq (Brazil), by CoNaCyT (Mexico), by Proyecto Basal FB 0821, CONICYT PIA ACT1413, Fondecyt 3170845 and 11130133 (Chile), by CONCYTEC (Consejo Nacional de Ciencia, Tecnología e Innovación Tecnológica), DGI-PUCP (Dirección de Gestión de la Investigación-Pontificia Universidad Católica del Peru), and VRI-UNI (Vice-Rectorate for Research of National University of Engineering) (Peru); by the Latin American Center for Physics (CLAF); and NCN Opus Grant No. 2016/21/B/ST2/01092 (Poland). We thank the MINOS Collaboration for use of its near detector data. Finally, we thank the staff of Fermilab for support of the beamline, the detector, and the computing infrastructure.
[1] K. Abe et al. (T2K Collaboration), Phys. Rev. Lett. 118, 151801 (2017).

[2] P. Adamson et al. (NOvA Collaboration), Phys. Rev. D 93, 051104 (2016).

[3] P. Adamson et al. (NOvA Collaboration), Phys. Rev. Lett. 116, 151806 (2016).

[4] R. Acciarri et al. (Fermilab Short Baseline Collaboration), arXiv:1503.01520.

[5] C. Adams et al. (LBNE Collaboration) arXiv:1307.7335.

[6] R. Acciarri et al. (DUNE Collaboration), arXiv:1512 .06148 .
[7] R. Wigmans, Calorimetry, International Series of Monographs on Physics (Oxford University Press, Oxford, England, 2017).

[8] R. Gran, M. Betancourt, M. Elkins, P. A. Rodrigues et al. (MINERvA Collaboration), Phys. Rev. Lett. 120, 221805 (2018).

[9] P. A. Rodrigues, J. Demgen, E. Miltenberger et al. (MINERvA Collaboration), Phys. Rev. Lett. 116, 071802 (2016).

[10] C. Andreopoulos et al., Nucl. Instrum. Methods Phys. Res., Sect. A 614, 87 (2010), Program version 2.8.4, with private modifications, used here. 
[11] S. Agostinelli et al., Nucl. Instrum. Methods Phys. Res., Sect. A 506, 250 (2003).

[12] J. Allison et al., IEEE Trans. Nucl. Sci. 53, 270 (2006).

[13] L. Aliaga et al. (MINERvA Collaboration), Nucl. Instrum. Methods Phys. Res., Sect. A 743, 130 (2014).

[14] L. Aliaga et al. (MINERvA Collaboration), Nucl. Instrum. Methods Phys. Res., Sect. A 789, 28 (2015).

[15] C. L. Cowan, F. Reines, F. B. Harrison, H. W. Kruse, and A. D. McGuire, Science 124, 103 (1956).

[16] J. F. Amsbaugh et al., Nucl. Instrum. Methods Phys. Res., Sect. A 579, 1054 (2007).

[17] H. Zhang et al. (Super-Kamiokande Collaboration), Astropart. Phys. 60, 41 (2015).

[18] A. R. Back et al. (ANNIE Collaboration), arXiv:1707.08222.

[19] J. F. Beacom and M. R. Vagins, Phys. Rev. Lett. 93, 171101 (2004).

[20] F. J. Hasert et al. (Gargamelle Neutrino Collaboration), Phys. Lett. B 46, 138 (1973).

[21] F. J. Hasert et al. (Gargamelle Neutrino Collaboration), Nucl. Phys. B73, 1 (1974).

[22] W. F. Fry and D. Haidt, Report No. CERN-75-01, 1975.

[23] A. Entenberg, J. Horstkotte, W. Kozanecki, A. K. Mann, C. Rubbia, J. Strait, L. Sulak, P. J. Wanderer, and H. H. Williams, Phys. Rev. Lett. 42, 1198 (1979).

[24] J. Horstkotte, A. Entenberg, R. S. Galik, A. K. Mann, H. H. Williams, W. Kozanecki, C. Rubbia, J. Strait, L. Sulak, and P. J. Wanderer, Phys. Rev. D 25, 2743 (1982).

[25] L. A. Ahrens et al., Phys. Rev. D 35, 785 (1987).

[26] A. A. Aguilar-Arevalo et al. (MiniBooNE Collaboration), Phys. Rev. D 82, 092005 (2010).

[27] D. Perevalov, Neutrino-nucleus neutral current elastic interactions measurement in MiniBooNE, Ph. D. thesis, Alabama University, Report No. FERMILAB-THESIS2009-47, 2009.

[28] D. Casper, Nucl. Phys. B, Proc. Suppl. 112, 161 (2002).

[29] R. Brun, F. Bruyant, M. Maire, A. C. McPherson, and P. Zanarini, Report No. CERN-DD-EE-84-1, 1987.

[30] C. Zeitnitz and T. Gabriel, Nucl. Instrum. Methods Phys. Res., Sect. A 349, 106 (1994).

[31] R. Acciarri et al. (ArgoNeuT Collaboration), Phys. Rev. D 99, 012002 (2019).

[32] T. T. Bhlen, F. Cerutti, M. P. W. Chin, A. Fass, A. Ferrari, P. G. Ortega, A. Mairani, P. R. Sala, G. Smirnov, and V. Vlachoudis, Nucl. Data Sheets 120, 211 (2014).

[33] A. Ferrari, P. R. Sala, A. Fasso, and J. Ranft, Report No. CERN-2005-010, 2005; Report No. SLAC-R-773; Report No. INFN-TC-05-11, 2005.

[34] A. Friedland and S. W. Li, Phys. Rev. D 99, 036009 (2019).

[35] D. G. Michael et al. (MINOS Collaboration), Nucl. Instrum. Methods Phys. Res., Sect. A 596, 190 (2008).

[36] P. Adamson et al., Nucl. Instrum. Methods Phys. Res., Sect. A 806, 279 (2016).

[37] L. Aliaga et al. (MINERvA Collaboration), Phys. Rev. D 94, 092005 (2016); 95, 039903(A) (2017).

[38] C. Alt et al. (NA49 Collaboration), Eur. Phys. J. C 49, 897 (2007).

[39] S. P. Denisov, S. V. Donskov, Yu. P. Gorin, R. N. Krasnokutsky, A. I. Petrukhin, Yu. D. Prokoshkin, and D. A. Stoyanova, Nucl. Phys. B61, 62 (1973).
[40] A. S. Carroll et al., Phys. Lett. B 80, 319 (1979).

[41] J. V. Allaby et al. (IHEP-CERN Collaboration), Phys. Lett. B 30, 500 (1969).

[42] J. Park et al. (MINERvA Collaboration), Phys. Rev. D 93, 112007 (2016).

[43] C. H. L. Smith, Phys. Rep. 3, 261 (1972).

[44] R. Bradford, A. Bodek, H. S. Budd, and J. Arrington, Nucl. Phys. B, Proc. Suppl. 159, 127 (2006).

[45] R. A. Smith and E. J. Moniz, Nucl. Phys. B43, 605 (1972).

[46] D. Rein and L. M. Sehgal, Ann. Phys. (N.Y.) 133, 79 (1981).

[47] A. Bodek, I. Park, and U.-K. Yang, Nucl. Phys. B, Proc. Suppl. 139, 113 (2005).

[48] T. Yang, C. Andreopoulos, H. Gallagher, K. Hoffmann, and P. Kehayias, Eur. Phys. J. C 63, 1 (2009).

[49] Z. Koba, H. B. Nielsen, and P. Olesen, Nucl. Phys. B40, 317 (1972).

[50] T. Sjostrand, S. Mrenna, and P. Z. Skands, J. High Energy Phys. 05 (2006) 026.

[51] J. Nieves, J. E. Amaro, and M. Valverde, Phys. Rev. C 70, 055503 (2004).

[52] R. Gran, J. Nieves, F. Sanchez, and M. V. Vacas, Phys. Rev. D 88, 113007 (2013).

[53] R. Gran, arXiv:1705.02932.

[54] M. Valverde, J. E. Amaro, and J. Nieves, Phys. Lett. B 638, 325 (2006).

[55] J. Nieves and J. E. Sobczyk, Ann. Phys. (Amsterdam) 383, 455 (2017).

[56] A. S. Meyer, M. Betancourt, R. Gran, and R.J. Hill, Phys. Rev. D 93, 113015 (2016).

[57] P. A. Rodrigues, C. Wilkinson, and K. S. McFarland, Eur. Phys. J. C 76, 474 (2016).

[58] C. Wilkinson, P. A. Rodrigues, S. L. Cartwright, L. F. Thompson, and K. S. McFarland, Phys. Rev. D 90, 112017 (2014).

[59] A. Higuera, A. Mislivec et al. (MINERvA Collaboration), Phys. Rev. Lett. 113, 261802 (2014).

[60] A. Mislivec, A. Higuera et al. (MINERvA Collaboration), Phys. Rev. D 97, 032014 (2018).

[61] C. Berger and L. Sehgal, Phys. Rev. D 79, 053003 (2009).

[62] D. Rein and L. M. Sehgal, Nucl. Phys. B223, 29 (1983).

[63] J. Nieves, I. Ruiz Simo, and M. V. Vacas, Phys. Rev. C 83, 045501 (2011).

[64] J. Schwehr, D. Cherdack, and R. Gran, arXiv:1601.02038.

[65] P. A. Rodrigues et al. (MINERvA Collaboration) (to be published).

[66] M. Betancourt, A. Ghosh, T. Walton et al. (MINERvA Collaboration), Phys. Rev. Lett. 119, 082001 (2017).

[67] O. Altinok, T. Le et al. (MINERvA Collaboration), Phys. Rev. D 96, 072003 (2017).

[68] C. E. Patrick et al. (MINERvA Collaboration), Phys. Rev. D 97, 052002 (2018).

[69] X.-G. Lu, M. Betancourt, T. Walton et al. (MINERvA Collaboration), Phys. Rev. Lett. 121, 022504 (2018).

[70] D. Ruterbories, K. Hurtado, J. Osta et al. (MINERvA Collaboration), Phys. Rev. D 99, 012004 (2019).

[71] The SAID, http://gwdac.phys.gwu.edu, website provides access to both fits, models and the associated databases.

[72] Y. Hayato, Nucl. Phys. B, Proc. Suppl. 112, 171 (2002).

[73] Y. Hayato, Acta Phys. Pol. B 40, 2477 (2009). 
[74] T. Golan, C. Juszczak, and J. T. Sobczyk, Phys. Rev. C 86, 015505 (2012).

[75] P. Stowell et al., J. Instrum. 12, P01016 (2017).

[76] F. Dietrich, E. Hartouni, S. Johnson, G. Schmid, R. Soltz, W. Abfalterer, R. Haight, L. Waters, A. Hanson, R. Finlay, and G. Blanpied, J. Nucl. Sci. Technol. 39, 269 (2002).

[77] J. J. H. Menet, E. E. Gross, J. J. Malanify, and A. Zucker, Phys. Rev. C 4, 1114 (1971).

[78] W. F. McGill, R. F. Carlson, T. H. Short, J. M. Cameron, J. R. Richardson, I. Slaus, W. T. H. Van Oers, J. W. Verba, D. J. Margaziotis, and P. Doherty, Phys. Rev. C 10, 2237 (1974).

[79] P. U. Renberg, D. F. Measday, M. Pepin, P. Schwaller, B. Favier, and C. Richard-Serre, Nucl. Phys. A183, 81 (1972).

[80] J. F. Dicello, G. J. Igo, and M. L. Roush, Phys. Rev. 157, 1001 (1967).

[81] C. I. Zanelli, P. P. Urone, J. L. Romero, F. P. Brady, M. L. Johnson, G. A. Needham, J. L. Ullmann, and D. L. Johnson, Phys. Rev. C 23, 1015 (1981).

[82] M. H. MacGregor, W. P. Ball, and R. Booth, Phys. Rev. 111, 1155 (1958).

[83] W. Bauhoff, Atom. Data Nucl. Data Tabl. 35, 429 (1986).

[84] D. Ashery, I. Navon, G. Azuelos, H. Walter, H. Pfeiffer, and F. Schlepütz, Phys. Rev. C 23, 2173 (1981).

[85] C. Wilkin, C. R. Cox, J. J. Domingo, K. Gabathuler, E. Pedroni, J. Rohlin, P. Schwaller, and N. W. Tanner, Nucl. Phys. B62, 61 (1973).

[86] A. S. Clough et al., Nucl. Phys. B76, 15 (1974).

[87] B. Allardyce, C. Batty, D. Baugh, E. Friedman, G. Heymann et al., Nucl. Phys. A209, 1 (1973).

[88] C. J. Gelderloos, J. T. Brack, M. D. Holcomb, M. V. Keilman, D. J. Mercer, R. J. Peterson, R. A. Ristinen, and A. Saunders, Phys. Rev. C 62, 024612 (2000).

[89] M. Ibaraki, M. Baba, T. Miura, T. Aqki, T. Hiroishi, H. Nakashima, S.-I. Meigo, and S. Tanaka, J. Nucl. Sci. Technol. 39, 405 (2002).

[90] W. Schimmerling, T. J. Devlin, W. W. Johnson, K. G. Vosburgh, and R. E. Mischke, Phys. Rev. C 7, 248 (1973).

[91] R. G. P. Voss and R. Wilson, Proc. R. Soc. A 236, 41 (1956).
[92] W. P. Abfalterer, F. B. Bateman, F. S. Dietrich, R. W. Finlay, R. C. Haight, and G. L. Morgan, Phys. Rev. C 63, 044608 (2001).

[93] B. Bhandari et al. (CAPTAIN Collaboration), arXiv:1903 .05276 .

[94] C. M. Marshall et al. (MINERvA Collaboration), Phys. Rev. D 94, 012002 (2016).

[95] L. Fields, J. Chvojka et al. (MINERvA Collaboration), Phys. Rev. Lett. 111, 022501 (2013).

[96] G. Fiorentini, D. Schmitz, P. Rodrigues et al. (MINERvA Collaboration), Phys. Rev. Lett. 111, 022502 (2013).

[97] T. Le, J. L. Palomino et al. (MINERvA Collaboration), Phys. Lett. B 749, 130 (2015).

[98] C. E. Wiegand, T. Elioff, W. B. Johnson, L. B. Auerbach, J. Lach, and T. Ypsilantis, Rev. Sci. Instrum. 33, 526 (1962).

[99] R. A. Cecil, B. D. Anderson, and R. Madey, Nucl. Instrum. Methods 161, 439 (1979).

[100] J. B. Birks, Proc. Phys. Soc. London Sect. A 64, 874 (1951).

[101] J. B. Birks, The Theory and Practice of Scintillation Counting (Pergamon Press, Oxford, England, 1964), https://doi.org/10.1016/C2013-0-01791-4.

[102] B. von Krosigk, L. Neumann, R. Nolte, S. Rottger, and K. Zuber, Eur. Phys. J. C 73, 2390 (2013).

[103] B. von Krosigk et al., Eur. Phys. J. C 76, 109 (2016).

[104] P. Adamson et al. (MINOS Collaboration), Phys. Rev. D 91, 012005 (2015).

[105] K. Abe et al. (T2K Collaboration), Phys. Rev. D 98, 032003 (2018).

[106] R. Subedi, R. Shneor, P. Monaghan, B. D. Anderson, K. Aniol et al., Science 320, 1476 (2008).

[107] R. Acciarri et al. (ArgoNeuT Collaboration), Phys. Rev. D 90, 012008 (2014).

[108] K. Partyka (ArgoNeuT Collaboration), AIP Conf. Proc. 1663, 080007 (2015).

[109] O. Palamara (ArgoNeuT Collaboration), J. Phys. Soc. Jpn. Conf. Proc. 12, 010017 (2016).

[110] C. Adams et al. (MicroBooNE Collaboration), Eur. Phys. J. C 79, 248 (2019). 